\title{
Name Uniqueness Predicts Career Choice and Career Achievement
}

\author{
Han-Wu-Shuang Bao ${ }^{1,2}$, Huajian Cai ${ }^{1,2}$, C. Nathan DeWall ${ }^{3}$, Ruolei Gu ${ }^{1,2}$, Jun Chen ${ }^{4}$, Yu L. L. Luo ${ }^{1,2^{*}}$ \\ ${ }^{1}$ Institute of Psychology, Chinese Academy of Sciences, Beijing 100101, China \\ ${ }^{2}$ Department of Psychology, University of Chinese Academy of Sciences, Beijing 100049, China \\ ${ }^{3}$ Department of Psychology, University of Kentucky, Lexington, KY 40506-0044, USA \\ ${ }^{4}$ Beijing Meiming Science and Technology Company, Beijing 100107, China
}

* Corresponding author: Yu L. L. Luo (luoy@psych.ac.cn).

\section{ORCID}

Han-Wu-Shuang Bao: 0000-0003-3043-710X

Yu L. L. Luo: 0000-0002-8595-5265

\section{Data and code availability}

Data, code, and online materials are available at https://osf.io/8syrc/ [publicly available after acceptance]. H.-W.-S.B developed an R package 'ChineseNames' for the Chinese name database, which is available at https://github.com/psychbruce/ChineseNames.

\section{Acknowledgements}

This research was supported by National Social Science Fund of China (17ZDA324) and CAS Key Laboratory of Behavioral Science, Institute of Psychology (Y5CX052003). We thank Jochen E. Gebauer, Junlin Chen, Yiping Xie, and Hao Zheng for their comments on early drafts, and Huanhua Lu, Kuang He, Kejie Yao, and Guangyao Zhang for their assistance with data collection.

\section{Author contributions}

H.-W.-S.B., H.C., and Y.L.L.L. designed the research. H.-W.-S.B. and Y.L.L.L. performed the research. H.W.-S.B. and J.C. contributed new analytic tools. H.-W.-S.B. and Y.L.L.L. analyzed the data. H.-W.-S.B., Y.L.L.L., C.N.D., R.G., and H.C. wrote the paper.

\section{Competing interests}

The authors declare no competing interests. One author (J.C.) works in a commercial company. He offered and should be responsible for the Chinese name database (used in Studies 1, 2, 3, 5, and 6) and the Chinese resume data (used in Study 3). However, he took no role in research design, data analysis, and manuscript preparation. All conclusions reported in this article are independent from any commercial company. 


\begin{abstract}
Do personal names relate to career choice and career achievement? If so, how and why? We propose that uniqueness (vs. conformity), a dimension crucial for identity construction, may associate name (personal identity) with career (vocational identity). By testing a broad range of names and jobs, we identified a phenomenon — the name-job uniqueness fit — and revealed its benefit to career success. Seven studies (total $N=7.38$ million) provided correlational, experimental, and real-world evidence for that holding more unique names (less used in the population) predicted choosing more unique jobs (less occupied in the workforce). The need for uniqueness partly explained the name-job uniqueness fit. Furthermore, better name-job uniqueness fit predicted higher career achievement. Importantly, the name-job relations remained positive when considering confounding factors (e.g., socioeconomic status) and alternative explanations (e.g., parental influences) and were replicated in the U.S., the U.K., and China (with some cross-cultural differences). Our findings provide a perspective on how and why personal names relate to career outcomes beyond past theories.
\end{abstract}

Keywords: career, name, uniqueness, person-job fit, achievement 


\section{Name Uniqueness Predicts Career Choice and Career Achievement}

Careers run through our lives. For this lifework, we all face what we choose to do (career choice) and how well we do (career achievement). These two vital issues have stimulated scientists' massive efforts to understand what predict career outcomes, including macro (e.g., social capital) ${ }^{1}$, meso (e.g., parents' occupations) ${ }^{2}$, and micro factors (e.g., personality traits and values) ${ }^{3}$. Beyond these well-documented factors, the public and scholars have also wondered for long if another factor-personal names—could predict the choice of certain careers and even associate with the success in these careers ${ }^{4-6}$. The current research provides correlational yet robust evidence for that holding more unique names can predict choosing more unique jobs and achieving higher success in such employment.

Over the past two decades, scientists have examined the relations between personal names and career outcomes. Regarding career choice, prior findings have shown that people tend to choose the jobs with titles similar to their names in spelling; for example, someone named Dennis is more likely to become a dentist ${ }^{7,8}$. Psychologists termed this phenomenon as the name-similarity effect and attributed it to 'implicit egotism' - people unconsciously prefer things associated with themselves ${ }^{7}$. Regarding career achievement, the literature has documented the advantages of easy-to-pronounce names for lawyers ${ }^{9}$, masculine names for legal and political careers ${ }^{10,11}$, alphabetically-earlier surnames for scientific careers ${ }^{12}$, and noble-meaning surnames for managerial careers ${ }^{13}$.

However, previous studies suffered from methodological, analytical, and theoretical limitations. First, follow-up studies using more rigorous methods revealed little support for 
the name-similarity effect ${ }^{14-16}$, alphabetical-order effect ${ }^{17,18}$, and noble-meaning effect $^{19}$.

Thus, whether those effects are replicable and generalizable is still debatable. Second, prior research had several methodological and analytical problems: (i) deducing causality from correlational evidence; (ii) not ruling out confounds such as baby-naming trend (birth-cohort effect), socioeconomic status (SES), and parents' characteristics; and (iii) sampling only a fraction of names and jobs with some specific spellings or meanings ${ }^{14-16,19,20}$. Third, although researchers provided some theoretical explanations for those name effects, the explanations are not sufficient and lack direct empirical test ${ }^{14,15}$. Therefore, existing evidence is far from conclusive, leaving the relation between name and career still an unsolved puzzle.

The current research aims to investigate whether people's names relate to their career choice and career achievement by using large samples and diverse methodologies and addressing as many confounding factors and alternative explanations as possible. We focus on a psychological dimension relevant to both names and jobs: uniqueness (vs. conformity). Uniqueness refers to how a person is different from others ${ }^{21}$. Everyone has a motive to be distinctive, namely, a 'need for uniqueness' $(\mathrm{NfU})^{22}$. NfU is a universal human motive that is essential for constructing personal identity ${ }^{23,24}$ and can manifest through naming practices. Indeed, parents from both Western and Eastern cultures have become more likely to give unique names to their children than before ${ }^{25-27}$, especially in recent decades, when many cultures are becoming more individualistic ${ }^{28}$ and $\operatorname{looser}^{29}$. In labor markets, individuals with more unique names — albeit sometimes unfavorably treated during job recruitment ${ }^{30,31}$ _ might have a better chance to stand out from their peers ${ }^{32,33}$. 
Besides names, uniqueness also characterizes careers. Based on Holland's classic model $^{34}$, a two-dimensional model has recently been developed to guide vocational choices. One dimension is 'Extroversion-Introversion' (jobs requiring interpersonal skills vs. selfdiscipline skills); the other is 'Conformity-Originality' (jobs demanding structure/conformity vs. creativity/uniqueness) ${ }^{35,36}$. Whereas some jobs feature conformity and structure (e.g., clerks, managers), others feature uniqueness and creativity (e.g., artists, researchers) ${ }^{34,36}$. Such uniqueness-demanding or 'creative' jobs are, in general, less frequently occupied in the workforce (i.e., objectively unique) $)^{37,38}$. Moreover, uniqueness has become highly valued in the 21 st-century competitive workplace because it implies standing out from the crowd $\mathrm{d}^{39}$.

We propose that people pursue a psychological fit or match between name (personal identity) and career (vocational identity) on the 'uniqueness-conformity' dimension. Such a pursuit of 'name-job fit' (rather than a rebellion against the match) is in line with a large body of research supporting the 'person-job fit' theory: people desire jobs whose environments match their personal attributes ${ }^{36,40}$. People seek person-job fit because a suitable job's opportunities or resources can satisfy their psychological needs ${ }^{41,42}$. In our case, a person with a unique name may have a greater need for uniqueness ${ }^{22,43}$, which may predict uniquenessseeking behaviours ${ }^{21,44}$, such as choosing a unique job. Furthermore, the person-job fit theory also indicates that, when starting a career, better person-job fit is associated with higher job satisfaction and greater career success ${ }^{36}$. For example, creative people prefer jobs offering creative opportunities ${ }^{45}$, and such a fit between a creative person and a creative job promotes the self-efficacy on creativity ${ }^{46}$. In contrast, a person-job misfit may relate to poorer 
outcomes $^{36,47}$. Hence, according to the person-job fit theory and prior findings relating name uniqueness to occupational status ${ }^{32,33}$, we expect that, if taking a unique job, unique-name holders would outperform their common-name counterparts.

In this research, we conducted seven studies and two supplementary studies (for an overview, see Table 1) to examine a potential match between name uniqueness and job uniqueness (termed the 'name-job uniqueness fit') and explore its potential benefit to career achievement. We proposed three hypotheses: $(H 1)$ people with unique names are more likely to occupy unique jobs; $(\mathrm{H} 2)$ need for uniqueness mediates the name-job uniqueness fit; and (H3) better name-job uniqueness fit predicts higher career achievement in unique jobs. First, we tested $H 1$ and $H 2$ through one survey (Study 1) and two parallel experiments (Study 2). Because it is almost impossible to randomly assign people names at birth and then test the effects of those names on their career outcomes, we tried to identify causation by employing experimental manipulations (Study 2) and ruling out possible alternative explanations. However, such attempts can barely mirror the way names work in real life. Being aware of this and the correlational nature of our studies, we refrained from causal inference. Instead, we went on to examine whether $H 1$ could generalize to real-world contexts by analyzing millions of resumes from China (Study 3), the U.S., and the U.K. (Study 4). Next, we sought to replicate $H 1$ and examine $H 3$ by analyzing large-scale job-hunting data (Study 5) and multi-source archival data (Study 6) from China. Finally, we tested the generalizability of $H 3$ in the U.S. and the U.K. (Study 7). 


\section{Results}

Study 1: Name uniqueness relates to the uniqueness of future jobs. To test $H 1$ and $H 2$, we recruited 672 Chinese undergraduates to complete an online survey. They reported the jobs they intended to choose after graduation and selected its category from an 18-category job taxonomy (covering the majority of jobs in China). The person-environment fit theory indicates that: (i) the correspondence between person and environment characteristics can occur on both subjective and objective levels; (ii) subjective fit and objective fit are two distinct facets of the fit construct, with only low-to-moderate correlations between the two ${ }^{36,40}$. Accordingly, we employed both objective and subjective measures to assess the uniqueness of participants' names and jobs. We expected to find a correlation between the objective measures for names and jobs as well as a correlation between the two subjective measures, but not necessarily a correlation between name and job indices of different levels.

Objective job uniqueness was computed by log-transforming the percentage of workers occupying each job category in the national workforce of China (Supplementary Method 1). To ensure a sense of anonymity, we did not directly ask participants to report their names; instead, we obtained their names from their public profiles on Alipay, which included every user's given name (but no surname). Objective name uniqueness was computed by logtransforming the percentage of each given-name character used in the Han Chinese population within the specific birth cohort of each participant (Supplementary Method 2). Participants rated subjective job uniqueness and subjective name uniqueness. To further test potential mediation effects and rule out alternative explanations, we measured the need for 
uniqueness (NfU), the sense of uniqueness (SoU), gender, age, expected highest education, and family SES (including family income, parents' education, and parents' occupations).

Objective and subjective name-job uniqueness fit. Objective and subjective indices of either name uniqueness or job uniqueness were positively correlated (name: $r_{\mathrm{obj}-\text { subj }}=0.32$ $[0.25,0.39], P<0.001$; job: $r_{\mathrm{obj}-\text { subj }}=0.25[0.17,0.32], P<0.001 ; 95 \%$ confidence intervals [CI] are presented in brackets throughout this article), indicating that objective uniqueness can be perceived by individuals. More important, objective name uniqueness was positively correlated with objective job uniqueness $(r=0.14[0.07,0.22], P<0.001)$, supporting the name-job uniqueness fit on an objective level; subjective name uniqueness was positively correlated with subjective job uniqueness $(r=0.18[0.10,0.25], P<0.001)$, supporting the name-job uniqueness fit on a subjective level. We did not find any significant name-job correlations across objective and subjective levels $\left(P_{\mathrm{S}}>0.05\right.$; Supplementary Table 2$)$. In line with the person-job fit theory ${ }^{36,40}$, these results indicated that the objective fit and the subjective fit reflected two distinct aspects of the name-job uniqueness fit and may function in separate ways. Therefore, we tested them separately in regression models.

In ordinary least squares (OLS) regression, we controlled for potential confounds. One set of confounds included three other features of given names: (i) character uniqueness in a contemporary Chinese corpus (i.e., not the uniqueness in naming practices), (ii) name valence (i.e., positivity of character meaning, rated by independent raters), and (iii) name gender (i.e., difference in proportions of a character used by males vs. females) (for details, see Supplementary Method 2). Another set of confounds involved family SES. Parents' 
education levels and occupations may influence their preference for common vs. unique baby names ${ }^{48}$ and their children's working opportunities ${ }^{2}$. Thus, family SES may serve as a third variable underlying the name-job uniqueness fit.

Results showed that, although the three indices of family SES correlated modestly with name and job uniqueness (Supplementary Table 2), they no longer predicted job uniqueness when all predictors were included (Table 2). Importantly, objective name uniqueness still positively predicted objective job uniqueness (standardized $\beta=0.158[0.052$, $\left.0.263], P=0.003, r_{\text {partial }}=0.11\right)$ and subjective name uniqueness still positively predicted subjective job uniqueness $\left(\beta=0.117[0.042,0.192], P=0.002, r_{\text {partial }}=0.12\right)$ (Table 2).

The mediating role of $\mathrm{NfU}$. As expected, NfU positively predicted both objective and subjective job uniqueness (Table 2, Models 2.1/2.2). Mediation analysis showed that NfU partly mediated the subjective name-job fit: $\beta_{\text {indirect }}($ standardized indirect effect $)=0.0625$ [0.0357, 0.0992] (bias-corrected 95\% CIs with 5,000 bootstrapped samples are reported for all indirect effects in this article). Notably, the direct effect of subjective name uniqueness was still significant, $\beta_{\text {direct }}=0.116$ [0.044, 0.187], $P=0.002$ (Table 2, Model 2.2). When controlling for family SES and other confounds (listed in Table 2, Model 3.2), the mediation effect of NfU remained significant $\left(\beta_{\text {indirect }}=0.0263[0.0045,0.0554]\right)$.

For the objective name-job fit, the mediation pathway through only NfU was not significant $\left(\beta_{\text {indirect }}=0.0042[-0.0047,0.0164]\right)$. Instead, we found a serial multiple mediation through two mediators: people with objectively more unique names perceived their names as more unique (i.e., subjective name uniqueness; the first mediator). This perception of name 
uniqueness, in turn, predicted higher NfU (the second mediator) and the intention of choosing objectively more unique jobs $\left(\beta_{\text {indirect }}=0.0090[0.0040,0.0174]\right)$. This serial mediation remained significant $\left(\beta_{\text {indirect }}=0.0026[0.0006,0.0068]\right)$ after controlling for all confounds. Additionally, SoU complemented and preceded — but did not outperform-NfU as a mediator for both objective and subjective name-job fit (see Supplementary Results).

Ruling out alternative explanations. Although family income, parents' education, and parents' occupations did not explain away the name-job uniqueness fit, there was another third variable — parents' NfU— that may make children's name uniqueness, NfU, and job uniqueness covary. This might take place through inheritance or parenting. For example, parents with higher NfU may give birth to children with higher NfU, choose more unique names for their children, and encourage their children's uniqueness-seeking behaviours such as pursuing unique jobs.

We conducted two supplementary surveys to address this alternative explanation. Results showed that parents' NfU was not related to children's NfU, SoU, name uniqueness, or job uniqueness $(r \mathrm{~s}<0.10, P \mathrm{~s}>0.40$; Supplementary Study $\mathrm{S} 1)$. Moreover, participants' NfU could hardly predict the uniqueness of the names they chose for their offspring $(\beta=$ $0.064, P=0.23)$ after controlling for their birth year (Supplementary Study S2). Therefore, the name-job uniqueness fit was not reducible to parents' NfU.

Study 2: Experiencing holding unique names predicts choosing unique jobs. Study 1 showed that undergraduates' name uniqueness and the uniqueness of their future jobs 
somewhat matched on both objective and subjective levels, albeit not across levels.

Furthermore, we found that NfU could partly explain the name-job uniqueness fit and ruled out several alternative explanations (e.g., family SES, parents' NfU). However, the results of a survey may arise from some omitted third variables unknown to us. To have a more rigorous examination of $H 1$ and $H 2$, Study 2 employed well-controlled experiments and tested whether people in unique-name (vs. common-name) conditions would have a higher NfU and be more likely to choose a unique (vs. common) job.

It is impractical and unethical to randomly assign people different names at birth and examine the influences of names for a lifetime. Hence, we sought to approximate such name effects by developing an experimental paradigm—-Life Simulation Game', where participants experienced having a new identity, holding a new given name (but still with their surname), and applying for a job based on the new identity (see details in Methods and Supplementary Methods). We randomly assigned participants either a common or a unique name and also showed them other personal information (e.g., educational background) that was kept the same across conditions. Next, we asked them to choose a job between 'Project Operation' (a common job, described as requiring 'clear operation concept and organizational planning', etc.) and 'Product Design' (a unique job, described as requiring ‘unique design concept and creative thinking', etc.). We matched many confounding features of the names and jobs between the unique- and common-name conditions (Supplementary Methods; Supplementary Table 3). Participants also reported NfU and SoU based on their new identity. We applied this paradigm in two parallel experiments, which were identical in all 
aspects except three variations of the stimuli: (i) Study $2 \mathrm{a}(N=578)$ set the major of the new identity as a popular major in China ('economy'), whereas Study $2 \mathrm{~b}(N=470)$ did not specify the major of the new identity; (ii) Study $2 b$ used a different set of given names to examine the generalizability of the name effect; and (iii) Study $2 b$ added a note 'no gender limitation' into the job descriptions to eliminate potential gender bias concerning the occupation.

The effect of name uniqueness on job choice. As the job choice was a dichotomous outcome variable, we performed a logistic regression with name-uniqueness manipulation $($ common $=-0.5$, unique $=0.5)$, participant gender $($ female $=-0.5$, male $=0.5 ;$ corresponding to the new identity's gender), and their interaction as predictors (we used effect coding to avoid multicollinearity between predictors). Supporting $H 1$, Study 2 a showed that participants assigned with unique (vs. common) names were more likely to choose the unique job $(38.5 \%$ vs. $27.9 \%): B=0.490, S E=0.180, P=0.006$, odds ratio $(\mathrm{OR})=1.63[1.15,2.32]$ (Table 3, Model 1.2). In addition, male participants preferred the unique job than did females $(B=0.417, S E=0.180, P=0.020, \mathrm{OR}=1.52[1.07,2.16])$, but gender did not moderate the effect of name manipulation $\left(B_{\text {interaction }}=0.094, S E=0.360, P=0.79\right)$. Study $2 \mathrm{~b}$ replicated the effect of holding unique (vs. common) names on choosing the unique job (55.6\% vs. $40.8 \%$ ): $B=0.597, S E=0.187, P=0.001, \mathrm{OR}=1.82[1.26,2.62]($ Table 3, Model 2.2). As we emphasized gender equality in Study $2 \mathrm{~b}$, neither gender $(B=0.199, S E=0.187, P=0.29)$ nor its interaction with name $\left(B_{\text {interaction }}=-0.064, S E=0.374, P=0.86\right)$ was significant.

Then, we combined the two datasets and conducted a logistic regression while controlling for all possible confounds (age, education, parents' education, and surname 
uniqueness). The name-uniqueness effect remained significant (44.6\% vs. $34.4 \%$ ): $B=0.429$, $S E=0.135, P=0.001, \mathrm{OR}=1.54[1.18,2.00]($ Table 3 , Model 4.2). There was an additional effect of study version. Participants in Study $2 b$ were more likely to choose the unique job (2b vs. $2 \mathrm{a}: 47.8 \%$ vs. $31.6 \%$ ): $B=0.688, S E=0.135, P<0.001, \mathrm{OR}=1.99[1.53,2.59]$. This was possibly due to that the major of the new identity was set as a popular, common one in Study 2a, implying an effect of having a common major on choosing a common job.

The mediating role of $N \boldsymbol{H}$. Based on the combined data, a 2 (name: unique vs. common $) \times 2($ gender $) \times 2$ (study: 2 a vs. 2 b) between-subjects analysis of variance $($ ANOVA) revealed a significant main effect of name-uniqueness manipulation on $\mathrm{NfU}(F(1,1040)=$ 21.78, $\left.P<0.001, \eta_{\mathrm{p}}^{2}=0.021\right)$. All the other main and interaction effects were not significant $\left(F \mathrm{~s} \leq 3.63, P \mathrm{~s} \geq 0.057, \eta_{\mathrm{p}}^{2} \mathrm{~s} \leq 0.003\right)$. As predicted, experiencing holding a unique (vs. common) name increased NfU: $M=3.49$ vs. $3.26, S D_{\text {pooled }}=0.79, t(1040)=4.67, P<0.001$, Cohen's $d=0.29[0.17,0.41]$. We found a similar result for SoU, but with a smaller effect size (see Supplementary Results). Moreover, NfU (rather than SoU and demographics) predicted a higher possibility to choose the unique job (Table 3, Model 4.2).

Then, we tested the mediating role of NfU while controlling for the effects of gender and study (Fig. 1). Replicating Study 1, NfU mediated the effect of holding unique names on choosing unique jobs: $B_{\text {indirect }}=0.1520[0.0842,0.2396]$ (unstandardized indirect effect and bootstrapped $95 \% \mathrm{CI})$. The direct effect of name uniqueness remained significant $\left(B_{\text {direct }}=\right.$ $0.4234[0.1610,0.6857], P=0.002)$. Furthermore, we found that SoU complemented and preceded — but did not outperform—NfU as a mediator (see Supplementary Results). 
Although Study 2 simulated the influences of names in real life, it took place in a hypothetical and simplistic context that barely mirrored the real world. Thus, we could not argue for a similar causal effect of name uniqueness on career choice in real life. Nonetheless, Study 2 helped us establish the internal validity of the name-job uniqueness fit by controlling for personal background, other name features, and other job features (e.g., income level, social status; see Supplementary Methods). Subsequent studies using big data would help us further establish the ecological validity of the name-job uniqueness fit in real-world contexts.

Study 3: Ecological validity of name-job uniqueness fit in China. In the following studies, we sought to replicate and extend the name-job uniqueness fit effect by testing a broader range of names and jobs in real labor markets. Evidence from large-scale real-world data (Studies 3-7) would overcome the lack of ecological validity in surveys (Study 1) and experiments (Study 2). In Study 3, we examined the relation between employees' names and job positions based on 2,480,269 resumes from China. Since both given names and surnames were available, we included four given-name variables (given-name uniqueness, character uniqueness in a contemporary Chinese corpus, name valence, name gender), two surname variables (surname uniqueness, alphabetical order of surname initial), and the full-name length (Supplementary Method 2). We classified jobs into the same 18 job categories by matching keywords and computed objective job uniqueness as in Study 1 (Supplementary Methods). The resume data also included individuals' gender, age, and education level. We implemented three analytic strategies: (i) estimating marginal group means of 
name uniqueness across jobs (job-level aggregation) and job uniqueness across names (namelevel aggregation); (ii) using machine learning to explore which variable(s) can predict job uniqueness (a data-driven exploratory approach); and (iii) using hierarchical linear modeling (HLM) to test whether name uniqueness can predict job uniqueness while controlling for all possible confounds (a theory-driven confirmatory approach). We employed HLM also to address a clustering situation of individuals sharing the same given name and surname.

As a Han Chinese given name usually consists of $1 \sim 2$ characters, we took additional steps to address multi-character given names. When performing job-level aggregation and individual-level machine-learning analysis, we averaged scores across characters for each given-name variable (we list reasons for this practice in Supplementary Method 2). When performing name-level aggregation and HLM name-level analysis, to have a more rigorous examination and check the robustness of results, we tested three subsets of the full sample by respectively focusing on (i) the single-character given names, (ii) the first characters of all given names, and (iii) the last characters of all given names.

Aggregate-level analysis. First, we estimated marginal group means of name uniqueness for all the 18 job categories while controlling for demographics. People in more unique jobs had more unique given names but not more unique surnames (Fig. 2). Next, we estimated marginal means of job uniqueness for $>1,000$ given-name characters and 477 surnames, respectively, with demographics included as covariates. We computed name-level partial correlation $\left(r_{\text {name-level }}\right)$ as an effect size. People with more unique given names tended to occupy more unique jobs, which was observed for either the 1,185 single-character given 
names $\left(r_{\text {name-level }}=0.14[0.09,0.20]\right)$, the 1,688 first characters of all given names $\left(r_{\text {name-level }}=\right.$ $0.16[0.11,0.21])$, or the 1,632 last characters of all given names $\left(r_{\text {name-level }}=0.11[0.06\right.$, $0.16]), P \mathrm{~s}<0.001$ (Fig. 3a-c). In contrast, surname uniqueness was not associated with job uniqueness $\left(r_{\text {name-level }}=0.05[-0.04,0.14], P=0.28\right.$; Fig. $\left.3 \mathrm{~d}\right)$.

CRF machine-learning analysis. In machine learning, we used the Conditional Random Forest (CRF) algorithm, which can (i) explore all possible linear/nonlinear relations between predictors and the outcome and (ii) identify 'relatively important' predictors with less analytical bias and less overfitting problems. Prediction accuracy was estimated by how accurately a trained CRF model could predict the outcome in a testing dataset $\left(R^{2}{ }_{\text {out-of-bag }}\right)$, which provides an unbiased estimate of model performance in the future. Variable importance was gauged by how well a predictor could exceed a threshold of random noise in predicting the outcome and was reported as rank order ${ }^{49}$ (see details in Supplementary Method 3).

To address the disproportion of workers between the 18 job categories, we replicated the $\mathrm{CRF}$ analysis on 10 randomly selected subsamples (each $n=10,000)$ with balanced group sizes across job categories. We averaged prediction accuracy and variable importance across the replications (Kendall's coefficient of concordance $W_{\mathrm{t}}=0.80$ ). Results showed that the CRF model had small-to-medium prediction accuracy $\left(R^{2}{ }_{\text {out-of-bag }}=5.8 \%\right)$. Among all predictors, given-name uniqueness was the third most important predictor of job uniqueness, whereas surname uniqueness was not informative (Fig. 4a).

HLM name-level analysis. Machine-learning models are 'black boxes' without clear formulas, making their results difficult to interpret. To complement this data-driven approach, 
we applied a theory-driven approach: HLM. In a large sample, different people are likely to share identical name characters. Also, the sample sizes of individuals sharing the same name would be disproportional across names. To address these situations, we used HLM, where individuals (level 1) could be nested within both given names and surnames (level 2).

As shown in the HLM analysis (Supplementary Table 5), given-name uniqueness positively predicted job uniqueness even when controlling for all confounds, regardless if we targeted the single-character given names (standardized HLM coefficient $\gamma=0.019[0.016$, $0.022], t(1180)=10.90, P<0.001)$, the first characters of all given names $(\gamma=0.010[0.007$, $0.012], t(1683)=7.87, P<0.001)$, or the last characters of all given names $(\gamma=0.014[0.011$, $0.016], t(1627)=11.38, P<0.001)$. For other features of names, only given-name valence (a more positive name) and full-name length (a longer name) reliably predicted job uniqueness $(P \mathrm{~s}<0.05)$, but with much smaller effect sizes; holding a unique surname did not predict choosing a unique job $\left(P_{\mathrm{S}}>0.40\right)$. The absence of surname effect in China is likely due to the small diversity and low salience of Chinese surnames ${ }^{50}$ (Supplementary Note 1).

Study 4: Ecological validity of name-job uniqueness fit in the U.S. and the U.K. Based on 2.48 million resumes from Chinese labor markets, Study 3 demonstrated an objective fit between name uniqueness and job uniqueness in the real world. Study 4 aimed to replicate it in Western cultures by analyzing large-scale resume data from the U.S. and the U.K.

Substantial evidence has demonstrated that the distinctiveness motive or NfU prevails in both Eastern and Western cultures ${ }^{23-28}$. As Studies 1 and 2 have shown that NfU serves to 
associate unique names with unique jobs, we expected that the name-job uniqueness fit would generalize to Western cultures.

From LinkedIn, we collected resumes of 881,099 U.S. employees (involving 1,501 given names and 1,130 surnames) and 149,405 U.K. employees (involving 585 given names and 604 surnames). We followed LinkedIn's classification of jobs (147 categories) to estimate objective job uniqueness (Supplementary Method 1). Name variables included the uniqueness, length, and alphabetical order of initials for both given names and surnames (Supplementary Method 2). Gender was the only available demographic variable.

Aggregate-level analysis. We estimated marginal means with gender controlled. Consistent with Study 3, both U.S. and U.K. employees in more unique jobs had more unique given names; but unlike Study 3, they also had more unique surnames (Supplementary Fig. 1). From another perspective, employees with more unique given names ( $r_{\text {name-level[U.S.] }}=0.23$ $\left.[0.19,0.28] ; r_{\text {name-level[U.K.] }}=0.23[0.15,0.31]\right)$ and more unique surnames $\left(r_{\text {name-level[U.S.] }}=\right.$ $\left.0.16[0.10,0.21] ; r_{\text {name-level[U.K. }]}=0.26[0.18,0.33]\right)$ tended to occupy more unique jobs $(P \mathrm{~s}<$ 0.001; Fig. 3e-h).

CRF machine-learning analysis. Following Study 3, we repeated the CRF analysis for 10 times (Kendall's $W_{\mathrm{t}}=0.89$ for U.S. and 0.83 for U.K.), each time with a randomly selected subsample of 10,000 cases with balanced group sizes across the 147 job categories. The CRF models for both countries had small-to-medium prediction accuracy $\left(R_{\text {out-of-bag }}^{2}=\right.$ $2.1 \%$ for U.S. and $7.0 \%$ for U.K.). Both given-name and surname uniqueness were relatively important predictors; surname uniqueness was even more critical than given-name 
uniqueness (Fig. 4b and 4d).

HLM name-level analysis. As machine learning is exploratory and cannot adjust for the influences of covariates $^{49}$, we conducted HLM analysis separately for U.S. and U.K. In HLM, individuals were clustered by given names and surnames, respectively; the six name variables and individuals' gender were predictors (Supplementary Table 6). Replicating Study 3, in both U.S. and U.K. samples, people with more unique given names occupied more unique jobs, which held after controlling for confounds: $\gamma_{\text {U.S. }}=0.034[0.030,0.039], t(1497)$ $=16.53, P<0.001 ; \gamma_{\text {U.K. }}=0.048[0.038,0.059], t(581)=8.78, P<0.001$. Unlike Study 3 , surname uniqueness also predicted job uniqueness: $\gamma_{\text {U.S. }}=0.026[0.023,0.028], t(1126)=$ $17.48, P<0.001 ; \gamma_{\text {U.K. }}=0.049[0.043,0.056], t(600)=14.91, P<0.001$. All the other name variables, except given-name length, were not predictive of job uniqueness.

Ruling out alternative explanations. While Study 1 showed that the name-job uniqueness fit was not reducible to family SES and parents' NfU, the finding of a positive correlation between surname uniqueness and job uniqueness could further partial out alternative explanations related to parents' psychological characteristics. Whereas given names are usually selected by parents after much consideration, surnames are always inherited from and seldom altered by parents. If parents' characteristics had confounded the relation between name uniqueness and job uniqueness, there would be a more substantial correlation for given names than for surnames. But Study 4 showed that surname uniqueness and given-name uniqueness had comparable correlations with job uniqueness. Hence, the name-job uniqueness fit was unlikely attributed to parents' personality (e.g., uniqueness), 
ability (e.g., creativity), or preference (e.g., a taste for eccentric stuff).

Additionally, as women in U.S. and U.K. usually take their husband's surname after marriage, there might be another alternative explanation that those women who had a higher NfU preferred both unique jobs and men with unique surnames. If this was the case, we would find a larger relation between surname uniqueness and job uniqueness among females than among males. However, HLM results for each gender (with other confounds controlled) did not support this possibility: surname uniqueness had comparable effects in predicting job uniqueness for males $\left(\gamma_{\text {U.S. }}=0.031[0.027,0.035], t(1123)=15.87, P<0.001 ; \gamma_{\text {U.K. }}=0.051\right.$ $[0.044,0.059], t(600)=12.99, P<0.001)$ and females $\left(\gamma_{\text {U.S. }}=0.018[0.014,0.022], t(1122)=\right.$ $\left.8.95, P<0.001 ; \gamma_{\text {U.K. }}=0.044[0.034,0.054], t(582)=8.86, P<0.001\right)$.

Explaining cultural differences. The inconsistent findings on surname uniqueness between Studies 3 and 4 may arise from cultural differences in the usage and salience of surnames. Surnames are often used alone in English-speaking countries, especially when speaking about professionals ${ }^{51}$. The higher salience of surnames in English-speaking countries plausibly explained its effects in U.S. and U.K. samples. Contrastingly, surnames are less salient and less diverse than given names in $\mathrm{China}^{50}$, which may result in the absence of surname effect in Chinese samples (Supplementary Note 1).

\section{Study 5: Name-job uniqueness fit and its potential benefit to career achievement among}

job hunters. Studies 1-4 consistently showed a match between name uniqueness and job uniqueness, which was evident across samples (students and employees), construct levels 
(objective and subjective), paradigms (correlational and experimental), outcomes (career planning and real job choice), and cultures (Eastern and Western). Moreover, while Studies 1-2 demonstrated its internal validity by addressing as many alternative explanations as possible, Studies 3-4 established its ecological validity in real labor markets. Studies 5-7 aimed to extend these findings by examining the consequences of the name-job uniqueness fit. Specifically, we tested if the more people's names and jobs fit on uniqueness, the higher they would achieve in careers (H3). In Studies 5 and 6, we also re-examined the name-job uniqueness fit $(H 1)$.

In Study 5, we collected 3,134,813 Chinese job hunters' public profiles from a jobhunting website in China: Ganji.com. With a structured classification of jobs, sufficient demographic information (Supplementary Table 7), and a proxy measure for career achievement (i.e., expected monthly salary), this large dataset permitted a more rigorous and comprehensive test of our hypotheses.

Effect \#1: Name-job uniqueness fit. The aggregate-level analysis, adjusting for all demographics, showed that job hunters with more unique given names tended to hunt for more unique jobs, either for the 1,267 single-character given names $\left(r_{\text {name-level }}=0.12[0.07\right.$, $0.18])$, the 1,769 first characters of all given names $\left(r_{\text {name-level }}=0.10[0.06,0.15]\right)$, or the 1,738 last characters of all given names $\left(r_{\text {name-level }}=0.10[0.06,0.15]\right), P_{\mathrm{s}}<0.001$ (Fig. 3i-k). Surname uniqueness was not related to job uniqueness $\left(r_{\text {name-level }}=-0.08[-0.16,0.01], P=\right.$ 0.10; Fig. 31). Then, the CRF machine-learning analysis (Kendall's $W_{\mathrm{t}}=0.88 ; R_{\text {out-of-bag }}^{2}=$ 9.3\%) again identified given-name uniqueness as a relatively important variable in predicting 
job uniqueness, whereas surname uniqueness was not informative (Fig. 4c). Consistently, the HLM analysis showed that unique-name holders were more likely to seek unique jobs, which held true after controlling for all available confounds (Supplementary Table 8). This pattern was replicated for the single-character subset $(\gamma=0.017[0.014,0.020], t(1262)=9.72, P<$ $0.001)$, the first-character subset $(\gamma=0.024[0.021,0.026], t(1764)=18.81, P<0.001)$, and the last-character subset $(\gamma=0.025[0.023,0.028], t(1733)=19.39, P<0.001)$. These results replicated the name-job uniqueness fit among job hunters, supporting $H 1$.

Effect \#2: Name-job uniqueness fit predicts potential career achievement. In HLM analysis, we included job hunters' expected salary as the outcome variable (i.e., a proxy for career achievement), both given-name and surname uniqueness as individual-level predictors (level 1), and job uniqueness as a job-level predictor (level 2). If the name-job fit is beneficial to potential career achievement, higher name uniqueness would predict higher expected salary for relatively unique jobs but not so for relatively common jobs. In other words, job uniqueness would moderate the relationship between name uniqueness and expected salary. As this is a cross-level moderation effect, we report unstandardized HLM coefficients while estimating its effect size via the $t$-to- $r$ transformation: $R^{2}$ job-level $=t^{2} /\left(t^{2}+d f\right)$.

The HLM analysis showed that given-name uniqueness positively predicted expected salary among job hunters (Supplementary Table 9). Furthermore, the effect of given-name uniqueness (level 1) was moderated by job uniqueness (level 2): $\gamma_{\text {moderation }}=0.0101[0.0018$, $0.0185], t(45)=2.44, P=0.019, R^{2}$ job-level $=11.7 \%($ Supplementary Table 9, Model 1$)$. This moderation effect remained significant when all available confounds were controlled: 
$\gamma_{\text {moderation }}=0.0076[0.0004,0.0149], t(45)=2.12, P=0.039, R^{2}$ job-level $=9.1 \%$ (Supplementary

Table 9, Model 3). Particularly, the positive relation between given-name uniqueness and expected salary increased as job uniqueness raised: low-unique jobs, $\gamma=0.008$ [0.002, 0.015], $t(45)=2.63, P=0.012$; medium-unique jobs, $\gamma=0.016[0.010,0.022], t(45)=5.01, P<$ 0.001 ; high-unique jobs, $\gamma=0.024[0.017,0.030], t(45)=7.40, P<0.001$. In contrast, neither surname uniqueness nor its interaction with job uniqueness could predict expected salary $(P \mathrm{~s}>0.20 ;$ Supplementary Table 9, Model 3). Overall, job hunters with more unique given names tended to expect higher salary in labor markets, especially for those seeking unique (vs. common) jobs, supporting $H 3$.

\section{Study 6: Replicating name-job uniqueness fit and its benefit to career achievement in}

China. To corroborate the findings of Studies 1-5, we conducted Study 6 to replicate both the name-job uniqueness fit and its benefit to career achievement. We collected large-scale archival data $(N=673,387)$ on individuals' full names and career achievement from various websites in China, involving five relatively unique jobs (researchers, musicians, painters, film actors, and film directors) and five relatively common jobs (doctors, managers, real-estate agents, domestic helpers, and cooks). For example, the $H$-index was collected to indicate researchers' career achievement ${ }^{52}$. We tested $H 1$ and $H 3$ by using meta-regression, a special case of meta-analysis that can test group-level moderators (Supplementary Methods).

Effect \#1: Name-job uniqueness fit. First, we focused on given names and performed meta-regression to compare the group means of name uniqueness across jobs (Supplementary 
Table 10), with job group ( $0=$ common, $1=$ unique $)$ as a job-level moderator. Supporting $H 1$, people in unique jobs had more unique given names $(M=2.72[2.59,2.84])$ than did those in common jobs $(M=2.42[2.26,2.57]): B_{\text {moderation }}=0.299[0.098,0.500], z=2.92, P=0.004$, $R^{2}$ job-level $=40.9 \%$ (proportion of heterogeneity explained by job group). To check the robustness, we replaced the dichotomous job group with the continuous job uniqueness as the job-level moderator and obtained a similar result: $B_{\text {moderation }}=0.134[0.017,0.251], z=2.24$, $P=0.025, R^{2}$ job-level $=26.9 \%$. These findings, from a meta-analytic approach, replicated the name-job uniqueness fit that has been observed throughout Studies 1-5.

Effect \#2: Name-job uniqueness fit predicts real career achievement. Having replicated the name-job uniqueness fit, we tested its benefit to career achievement. We estimated the correlation between given-name uniqueness and career-achievement indices for each job and conducted meta-regression to compare the correlations between unique jobs and common jobs. Supporting $H 3$, given-name uniqueness correlated positively with career achievement for all unique jobs ( $r$ s: 0.03 0.17; mean $r=0.10[0.07,0.14], P<0.001)$ but not for common jobs $(r \mathrm{~s}:-0.06 \sim 0.05$; mean $r=-0.01[-0.05,0.03], P=0.63): B_{\text {moderation }}=0.113$ $[0.058,0.167], z=4.05, P<0.001, R^{2}$ job-level $=62.4 \%$ (Fig. 5). Using the continuous job uniqueness as the job-level moderator yielded a similar result: $B_{\text {moderation }}=0.055[0.027$, 0.083], $z=3.88, P<0.001, R^{2}$ job-level $=61.4 \%$.

For each job, we additionally performed OLS regression to evaluate the relation between name uniqueness and career achievement while controlling for all available confounds (Supplementary Tables 12 and 13). These results further supported the positive 
relation between given-name uniqueness and career achievement in unique jobs — but not in any common job (except for doctors). Besides given names, we also found that surname uniqueness correlated positively with career achievement for researchers, especially for social scientists than for natural scientists (Supplementary Results; Supplementary Table 13).

Study 7: Replicating the benefit of name-job uniqueness fit in the U.S. and the U.K. In the last study, we collected archival data from U.S. and U.K. to examine whether the relation between name uniqueness and career achievement in unique jobs could generalize to Western cultures. Limited by data availability, we sampled only one common job (managers) and two unique jobs (film directors and researchers) $(N=45,297$ for U.S. and 12,494 for U.K.). This limitation made the meta-regression analysis not applicable, so we report results separately for each job. First, for managers, none of the name variables significantly predicted their achievement indexed by 'log total net worth' $(P \mathrm{~s}>0.10$; Supplementary Tables 14 and 15).

Second, for film directors, their achievement quantified by 'mean movie rating' manifested no simple correlations with either given-name uniqueness $\left(r_{\text {U.S. }}=0.02[-0.01\right.$, $\left.0.06], P=0.22 ; r_{\text {U.K. }}=0.10[0.00,0.19], P=0.058\right)$ or surname uniqueness $\left(r_{\text {U.S. }}=0.02[-\right.$ $\left.0.02,0.06], P=0.33 ; r_{\text {U.K. }}=0.08[-0.02,0.17], P=0.14\right)$. But after controlling for confounds including gender and age, given-name uniqueness positively predicted film directors' achievement for both countries $\left(\beta_{\mathrm{U} . \mathrm{S}}=0.042[0.002,0.082], P=0.042 ; \beta_{\mathrm{U} . \mathrm{K} .}=0.128[0.028\right.$, 0.227], $P=0.012$; Supplementary Tables 14 and 15). This may result from two conflicting trends: Americans' given names became more unique from $1880 \mathrm{~s}$ to $2000 \mathrm{~s}^{25}$, whereas 
younger directors, who were more likely to have unique names, tended to receive lower ratings for their movies (Supplementary Tables 14 and 15). Hence, film directors' given-name uniqueness could predict their movie ratings after partialling out age differences.

Finally, for researchers, surname uniqueness was positively correlated with the $H$ index for both natural scientists $\left(r_{\mathrm{U} . S .}=0.14[0.13,0.15] ; r_{\mathrm{U} . \mathrm{K} .}=0.07[0.05,0.09]\right)$ and social scientists $\left(r_{\mathrm{U} . \mathrm{S}}=0.13[0.11,0.15] ; r_{\mathrm{U} . \mathrm{K} .}=0.06[0.02,0.09]\right), P_{\mathrm{S}}<0.005$. Unexpectedly, we found negative correlations between given-name uniqueness and the $H$-index for natural scientists $\left(r_{\text {U.S. }}=-0.16[-0.17,-0.15] ; r_{\text {U.K. }}=-0.12[-0.14,-0.10]\right)$ and social scientists $\left(r_{\text {U.S. }}\right.$. $\left.=-0.14[-0.16,-0.12] ; r_{\mathrm{U} . \mathrm{K} .}=-0.12[-0.15,-0.08]\right), P \mathrm{~s}<0.001$. OLS regression analyses controlling for confounds yielded similar results (Supplementary Tables 14 and 15). These findings may result from the higher salience and more frequent exposure of surnames than given names in academic domains, especially in publication and citation ${ }^{12,51}$ (for more discussion on the unexpected negative effects of given names, see Supplementary Note 2).

Overall, despite some inconsistent findings, Study 7 provided further support for $H 3$ in Western cultures by showing positive relations (i) between given-name uniqueness and career achievement among film directors and (ii) between surname uniqueness and career achievement among researchers. Like Study 4, the finding of surname uniqueness as a predictor of career outcomes could rule out several alternative explanations, such as parents' psychological characteristics and family SES, although the limited data sources did not permit addressing more alternative accounts. 


\section{Discussion}

Career choice and career success are two crucial issues in people's professional life.

Whether and how personal names (including given names and surnames) predict career choice and career achievement have intrigued the public for decades but are still debatable among academics. Across seven studies using diverse methods and samples (total $N=7.38$ million), we examined the phenomenon, mechanisms, and consequences of a novel effect: the name-job uniqueness fit. Three main findings emerged. First, people with more unique names tend to occupy more unique jobs, which has internal validity (Studies 1-2), ecological validity (Studies 3-6), and cross-cultural generalizability (Study 4). Second, NfU partly explains the name-job uniqueness fit, which is supported by both survey and experiment methods (Studies 1-2). Third, the name-job uniqueness fit has real-life benefits, which is replicated in large-scale field data from U.S., U.K., and China. In unique jobs, having a unique name predicts earning more money, winning more favor, and achieving better performance (Studies 5-7).

These findings remained significant while controlling for various confounds (e.g., birth cohorts, gender, education) and could not be explained away by several alternative explanations (e.g., parental influences). In particular, because parents' SES and NfU may relate to giving children unique names ${ }^{27,48}$, we focused on the two factors. Presumably, parents with higher NfU or SES may favor uncommon baby names ${ }^{27,48}$, give birth to children who naturally have a higher NfU or prefer unusual things, and provide greater support to help their children stand out in work ${ }^{2}$. We acknowledge these possibilities. However, they cannot 
fully explain what we have found in this research. First, in the Chinese samples, given-name uniqueness remained an independent predictor of choice of unique jobs when we controlled for parents' education, occupation, income, and other confounds. Second, Supplementary Studies S1 and S2 showed that parents' NfU did not significantly correlate with children's name uniqueness, job uniqueness, NfU, or SoU, especially when their birth year was considered. Third, since parents' characteristics rarely influence their children's surnames, the positive relations of surname uniqueness with choice of unique jobs (among the U.S. and U.K. samples) and achievement in unique jobs (researchers' $H$-index across the Chinese, U.S., and U.K. samples) further ruled out parents' motive (e.g., NfU) or capacity (e.g., SES) as alternative explanations to our results. Nonetheless, there may be some other candidates of alternatives that have not been addressed here and warrant future studies.

It is also worth noting that the current findings are correlational. Although we tried to approximate the causality through two experiments in Study 2, these experiments were conducted in an artificial context and made participants explicitly attend to their new names, which may increase the effect of name uniqueness on job choice. Therefore, we interpreted these results with caution and did not argue for any causal effect of name uniqueness on career outcomes in real life.

Nevertheless, our correlational but robust findings have implications for research on names, self-identity, and vocational behaviour. First, by targeting the uniqueness dimension, we have included a comprehensive range of names (vs. specific spellings or meanings) and jobs (vs. a limited and biased set) and replicated the findings across multiple cultures (vs. in a 
single culture), thus overcoming methodological limitations in previous name research. This dimensional approach outperforms previous research by permitting more inclusive sampling, more rigorous examination, and more generalizable conclusion. Connecting with early criticisms on the name research ${ }^{14,15,19,20}$, we have ruled out many confounds and alternative explanations, verified a theoretical account by empirical tests, and revealed both crosscultural consistency and cultural differences. Our endeavors demonstrate that the relations between names and real-life outcomes, at least some of them, are not spurious.

Second, the finding of the name-job uniqueness fit enriches our knowledge about identity construction. Psychologists have proposed that being unique or distinctive from others (i.e., the distinctiveness motive) plays a fundamental role in establishing a meaningful sense of identity ${ }^{23,24}$. But it remains unclear how such an identity process takes place in real life. Our studies imply a possible way: early in life, some people may derive a sense of unique identity from their relatively unique names; accompanying (or after) the emergence of this preliminary sense of unique identity, a corresponding motive - the distinctiveness motive - may grow in them and give rise to their NfU; then, driven by this motive or to satisfy NfU, they may pursue a unique job, which can consolidate their self-uniqueness. In this way, people can construct and maintain a unique identity by matching their unique names with unique jobs; otherwise, a mismatch might disrupt the consistency or integrity within one's self-identity ${ }^{23}$.

Our findings also shed light on the scope, mechanisms, and consequences of personjob fit. Previous work has indicated that many people desire a job that aligns with their 
personal identity, especially personality traits and values ${ }^{3}$; and such a person-job fit can help people satisfy their psychological needs ${ }^{41,42}$ and obtain better career outcomes ${ }^{36,47}$. Our studies extend this line of research in three ways: (i) highlighting name uniqueness as another component of personal identity that functions in person-job fit; (ii) demonstrating NfU as a possible mechanism underlying person-job fit; and (iii) providing large-scale, real-world, and multi-cultural correlational evidence for the beneficial consequences of person-job fit.

We hope this research can spark more investigation of the relationships between name uniqueness and human behaviours as well as the mechanisms and consequences of such relationships. First, although we uncovered one mechanism-NfU-underlying the name-job uniqueness fit, future research can explore other possibilities. For instance, prior research revealed that people hold stereotypes about how individuals with certain names should look like and those individuals may also self-fulfill such stereotypes by shaping their facial appearance ${ }^{53}$. Hence, it is plausible to expect that unique-named people may be perceived as more suitable for unique jobs, which may further facilitate them to self-fulfill others' expectations. Second, we found that the name-job uniqueness fit existed in both the East and the West, although how this fit was implemented varied across cultures to some extent. This corresponds to the fact that the distinctiveness motive is prevalent across individualistic and collectivistic cultures, but it is realized in different ways in different cultures ${ }^{24}$. The previous and present findings together suggest culture as a moderator of the connections between name uniqueness and human behaviours, thus warranting future studies to test the moderating role of culture and identify other boundary conditions of the name effects. Third, from a 
broader perspective of person-environment fit $^{36,40}$, follow-up studies may test the effects of name uniqueness in domains beyond vocational behaviour. One possibility is close relationships and marriage. Is there also a fit between partners' name uniqueness in mate selection? Does such a fit relate to greater relationship satisfaction?

Finally, we draw these conclusions with caution. Many people desire a suitable and successful career. Career choice and career achievement can be predicted by numerous socioeconomic and individual-difference factors ${ }^{1-3}$. Name is just one of them, easy to be neglected but predictive. However, one should not view the name-job uniqueness fit as a support for 'nominative determinism' (a lay belief that names can 'determine' careers) ${ }^{4-6,8,16}$. In fact, no single factor can sufficiently cause one's career outcomes by itself, neither one's name nor one's family. We hope that people looking for career guidance can get inspiration from our findings, without doubting the influence of their free will on their career trajectory. 


\section{Methods}

Details of common methods across studies (e.g., computation of name variables) and specific methods within each study are described in Supplementary Methods. All studies were approved by the Institutional Review Board at the Institute of Psychology, Chinese Academy of Sciences (H19042). For surveys (Study 1) and experiments (Study 2), all participants provided online informed consent. For big data collected from the Internet (Studies 3-7), we collected publicly available data by using web crawlers (programmed by R or Python) while strictly following Chinese laws and the crawling policy of each website.

Study 1. Participants. A power analysis indicated that 652 participants were required to detect a small effect $\left(f^{2}=0.02\right)$ with two-tailed $\alpha=0.05$ and power $=95 \%$ in OLS regression. We finally had 672 undergraduates with real names available (343 females; $M_{\text {age }}=20.45 \pm$ 1.97 years [mean \pm standard deviation]). Specifically, 101 were recruited from a university in Beijing and 571 were from the participant pool of an online-survey platform in China (WJX.cn, https://www.wjx.cn/).

To obtain participants' real given names, we asked them to provide their Alipay account at the end of the survey (i.e., an e-mail address or a phone number), through which we paid them compensation. Alipay is a popular e-payment platform in China and requires users to register with real names. Users can decide whether to make their given names publicly visible (but surnames are always kept private). As some participants may not show their profile to the public or may even not have an Alipay account, we first recruited 745 
participants and then excluded 51 whose Alipay profiles were not visible and 22 whose gender reported in the survey was inconsistent with that in their Alipay account.

Job uniqueness. We assessed job uniqueness with both objective and subjective measures. Participants first reported the job they would take after graduation: 'Please write down the job that you will be most likely to take for a long time in the future, with many reallife conditions considered altogether.' Then, they selected the category of the job according to an 18-category job taxonomy adapted from the 2010 China census (Supplementary Method 1). The order of the 18 categories in the option list was randomized across participants. To help them select, we provided four exemplars for each category. Based on the selected categories and the statistics of national workforce, we obtained objective job uniqueness (Supplementary Table 1). Based on participants' evaluation on the uniqueness of their future jobs $(0=$ very common, $10=$ very unique $)$, we obtained subjective job uniqueness.

Given-name uniqueness. Based on a Chinese name database, we computed objective name uniqueness of each participant's real given name, adjusting for their birth cohort; for multi-character given names, we computed the mean score across characters (Supplementary Method 2). Participants also rated the uniqueness of their given names $(1=$ very common, $9=$ very unique), constituting a subjective index.

Need for uniqueness (NfU). We used the 4-item Self-Attributed Need for Uniqueness Scale (e.g., 'I prefer being different from other people') ${ }^{44}$ to measure participants' NfU (1= strongly disagree, $5=$ strongly agree; Cronbach's $\alpha=.82$ ).

Sense of uniqueness (SoU). We used the 5-item Personal Sense of Uniqueness Scale 
(e.g., 'I feel unique' $)^{54}$ to measure participants' SoU $(1=$ strongly disagree, $5=$ strongly agree; Cronbach's $\alpha=.77)$.

Demographic and parental information. Participants reported their gender, age, expected highest level of education $(1=$ high school or below, $2=$ junior college, $3=$ bachelor, 4 = master , 5 =doctor $)$, and family SES which includes household income $(1=$ lowest level, $5=$ medium level, $9=$ highest level in the society) and both parents' education (using the same 5-point scale as above) and jobs (selected from the same 18 job categories). Fathers' and mothers' scores were averaged to compute parents' education and parent's objective job uniqueness.

Study 2. Participants. A power analysis recommended 470 580 participants to detect a small-to-medium effect (Cohen's $d=0.30$ ) with $\alpha=0.05$ and power $=90 \sim 95 \%$. We recruited 578 undergraduates in Study 2a (289 females; $M_{\text {age }}=20.71 \pm 1.76$ years $)$ and 470 in Study $2 b$ (235 females; $M_{\text {age }}=20.86 \pm 1.64$ years). To note, we first recruited participants from the online participant pool of WJX.cn; to balance the gender ratio, we further recruited male participants from two Chinese social-networking platforms (QQ and WeChat) and a university in Beijing, China.

Experimental procedure. The experiment was conducted online and entitled the 'Life Simulation Game'. For ethical concern, we mentioned to participants that their choices in this task would not influence their real life. Participants first provided their demographic information (gender, age, education, and parents' education). Then, in sequence, they (i) 
provided their surname; (ii) randomly received a common or a unique new given name, along with other background information; (iii) typed down the new full name in a blank; (iv) were instructed to apply for a job position ('Project Operation' vs. 'Product Design') completely based on their new identity; and (v) indicated to what extent they made the decision based on their new identity ( 1 = not at all, $6=$ completely based on the new identity).

Participants who did not identify with the new identity, i.e., whose rating $\leq 3$ in the step (v), could not continue the experiment but were directed to the end and debriefed. Since the WJX.cn does not support downloading unqualified data, the invalid cases could not be analyzed. If passing this item (i.e., rating $\geq 4$ ), participants then completed the same measures of SoU and NfU as in Study 1—still based on their new identity. Afterwards, participants were notified that the game was over and they could forget the new identity. To preserve anonymity, we did not collect participants' own given names.

Experimental materials. We manipulated name uniqueness by randomly assigning participants either a common or a unique given name. Importantly, participants were not told whether the name was common or unique. All names were single characters that varied only on uniqueness, with many confounding features matched (e.g., name valence; Supplementary Methods). Specifically, common vs. unique names were ‘鹏(peng2) vs. 骢(cong1)’ for male and ‘婷(ting2) vs. 活(tian2)’ for female in Study 2a, and ‘涛(tao1) vs. 㥷(geng1)’ for male and ‘燕(yan4) vs. 莼(chun2)’ for female in Study 2b (Supplementary Table 3).

Surname uniqueness. Participants reported their real surnames, whose percentage of usage in the Han Chinese population was log-transformed to get an objective index of 
surname uniqueness (Supplementary Method 2).

Study 3. Sample. We collaborated with Beijing Qingyu Information Technology Company to obtain 2,735,344 resumes of Chinese workers. This company collected public resumes from a variety of Chinese professional networking websites (similar to LinkedIn) in 2015. Personal sensitive information (e.g., phone number) was not collected. Each resume included full name (most were real names), gender, age, education, and current job position.

For data cleaning, we step-by-step excluded 182,419 invalid cases (e.g., without a real name, doing a part-time job), 30,677 duplicate cases (matched by name, gender, age, education, and job), and 41,979 cases whose job information was ambiguous. Finally, we obtained a sample of 2,480,269 resumes $\left(35.0 \%\right.$ females; age range: $18 \sim 70$ years, $M_{\text {age }}=$ $32.34 \pm 6.09$ years; education: $10.6 \%$ high school or below, $27.0 \%$ junior college, $52.4 \%$ bachelor, $9.4 \%$ master, and $0.6 \%$ doctor [coded as 1 to 5 , respectively]).

In HLM analysis, to satisfy the minimum sample-size requirement and obtain reliable results, we excluded given names and surnames shared by $<30$ individuals ${ }^{55}$. Even so, there were still sufficient unique names in this large dataset.

Variables. We computed objective job uniqueness based on the 18 job categories in the same way as in Study 1 (Supplementary Method 1; Supplementary Table 1). Name variables included (i) given-name uniqueness, valence, and gender; (ii) the uniqueness of given-name character in a contemporary Chinese corpus; (iii) surname uniqueness and initial; and (iv) full-name length (Supplementary Method 2). The resumes included only three 
demographic variables - gender, age, and education.

Study 4. Sample. We collected public resumes from LinkedIn by searching English names combined by 10,000 given names and 1,000 surnames (Supplementary Methods). We initially collected 910,943 resumes of U.S. users and 163,435 resumes of U.K. users. For data cleaning, we excluded cases without valid names or jobs and duplicate cases (matched by given name, surname, gender, nationality, and job). Following Study 3, we also excluded given names and surnames shared by $<30$ individuals ${ }^{55}$. Finally, we had 881,099 resumes from U.S. (45.8\% females) and 149,405 resumes from U.K. (38.4\% females).

Variables. We computed objective job uniqueness based on LinkedIn's 147 job categories separately for the U.S. and U.K. samples (Supplementary Method 1). For name variables, we considered both given names and surnames and measured their uniqueness, length (i.e., number of letters), and alphabetical order of initials (Supplementary Method 2). Since birth year was not available in the resumes, we inferred a possible range of birth years of LinkedIn users (1940 1990) and computed given-name uniqueness by averaging the percentages of each name in the English name database across this range. Gender was the only available demographic variable.

Study 5. Sample. We collected public resumes of job hunters from a job-hunting website in China-Ganji.com (http://www.ganji.com/qiuzhi/). Ganji.com classifies jobs into 47 categories and organizes resumes by city. We sampled 50 cities for a representative sample 
and initially collected 5,512,224 resumes (Supplementary Methods). Each resume included full name, gender, age, education, working experience, expected monthly salary, and expected job position (for descriptive statistics, see Supplementary Table 7).

For data cleaning, we step-by-step excluded 584,875 cases without real names (detected by the same criteria as in Study 3), 610,429 duplicate cases (matched by name, gender, age, education, job, and city), and 1,182,107 cases without expected salary, education, or working-experience information. Finally, we obtained 3,134,813 resumes (42.8\% females; age range: $16 \sim 70$ years, $M_{\text {age }}=27.80 \pm 7.78$ years). Following Studies 3 and 4, we excluded given names and surnames shared by $<30$ individuals ${ }^{55}$ in HLM.

Variables. To compute objective job uniqueness and make it comparable to Study 3 , we recoded the 47 job categories classified by Ganji.com into the same 18 categories as used in Study 3 (see details in Supplementary Methods and Online Materials). We included the same set of name variables as in Study 3.

Achievement index. Though not the best indicator of career achievement, the 'expected monthly salary' can indicate people's income in the future. Thus, we used it as a proxy measure for career achievement. To note, in order to adjust for city-level variation in salary, we included random intercepts of city in HLM (Supplementary Table 9).

Study 6. Sample. We searched for as many web sources as possible that kept public records of individual full names and reliable indicators of career achievement. According to objective job uniqueness (Supplementary Table 1), we ended up with ten websites covering five 
relatively common and five relatively unique jobs. Data for common jobs involved 472,751 doctors (from haodf.com), 367 managers (from forbeschina.com), 31,576 real-estate agents (from lianjia.com), 55,807 domestic helpers (from bm.haobangni.com), and 280 cooks (from zhchushi.com). Data for unique jobs involved 88,152 natural-science and 13,751 socialscience researchers (from xueshu.baidu.com), 3,988 musicians (from y.qq.com), 1,037 painters (from huajia.cc), 736 film actors (from movie.mtime.com), and 3,716/1,226 film directors (from movie.douban.com and movie.mtime.com, respectively). For details, see Supplementary Methods and Supplementary Table 10.

Name variables. We computed given-name and surname uniqueness by using the same formulas as in former studies (Supplementary Method 2). Yet, for data sources without birth-year information (see Supplementary Table 10), we computed given-name uniqueness by taking the mean percentage of a character across all available birth cohorts in the Chinese name database.

Achievement indices. To make the indices of career achievement comparable across occupations, we classified them into three types: (i) income, including 'log monthly salary' (for domestic helpers), 'log total net worth' (for managers), and 'log payment for pictures' (for painters); (ii) liking, including 'mean favor rating' (for film actors), 'mean movie rating' (for film directors), and 'mean patients' rating' (for doctors); and (iii) performance, including 'log total deals' (for real-estate agents), 'log total followers' (for musicians), 'star ranking' (for cooks), and the ' $H$-index' (for researchers) $)^{52}$. 
Study 7. Sample. Similar to Study 6, we collected archival data with both full names and achievement indices for unique and common jobs from the U.S. and the U.K. In line with Study 6 and previous research ${ }^{37,38}$, we accessed data for one common job, managers $\left(N_{\text {U.S. }}=\right.$ 525 and $N_{\text {U.K. }}=41$ from forbes.com $)$, and two unique jobs, researchers $\left(N_{\text {U.S. }}=31,988\right.$ and $N_{\mathrm{U} . \mathrm{K} .}=9,397$ natural scientists, $N_{\mathrm{U} . \mathrm{S} .}=10,275$ and $N_{\mathrm{U} . \mathrm{K} .}=2,670$ social scientists, from Google Scholar, scholar.google.com $)$ and film directors $\left(N_{\mathrm{U} . \mathrm{S} .}=2,509\right.$ and $N_{\mathrm{U} . \mathrm{K} .}=386$ from

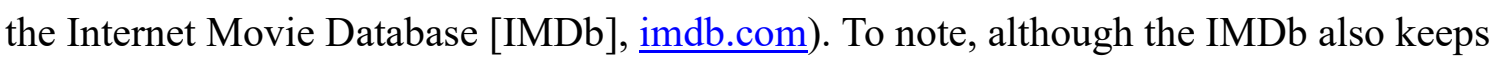
records of film actors, it does not provide a reliable index of their achievement. The only available index was a rank order of page view indicating actors' weekly popularity (e.g., 'Top 500'), which was apparently not a reliable measure of their career achievement. Therefore, we did not include film actors as a test case.

Name variables. We included the same set of name variables as in Study 4.

Achievement indices. Similar to Study 6, we adopted three types of achievement indices: income ('log total net worth' for managers), liking ('mean movie rating' for film directors), and performance (the ' $H$-index' for researchers). 


\section{References}

1. Ng, T. W. H., Eby, L. T., Sorensen, K. L., \& Feldman, D. C. (2005). Predictors of objective and subjective career success: A meta-analysis. Personnel Psychology, 58(2), 367-408.

2. Hout, M. (2018). Americans' occupational status reflects the status of both of their parents. Proceedings of the National Academy of Sciences, 115(38), 9527-9532.

3. Kern, M. L., McCarthy, P. X., Chakrabarty, D., \& Rizoiu, M.-A. (2019). Social media-predicted personality traits and values can help match people to their ideal jobs. Proceedings of the National Academy of Sciences, 116(52), 26459-26464.

4. New Scientist. (1994, December 17). Feedback. https://www.newscientist.com/article/mg14419566-600feedback/

5. Butcher, J. (2003). Giving in to the name game. The Lancet, 361(9360), 886.

6. BBC Capital. (2018, April 5). Do our names push us towards certain jobs? http://www.bbc.com/capital/story/20180404-do-our-names-push-us-toward-certain-jobs

7. Pelham, B. W., Mirenberg, M. C., \& Jones, J. T. (2002). Why Susie sells seashells by the seashore: Implicit egotism and major life decisions. Journal of Personality and Social Psychology, 82(4), 469-487.

8. Limb, C., Limb, R., Limb, C., \& Limb, D. (2015). Nominative determinism in hospital medicine. The Bulletin of the Royal College of Surgeons of England, 97(1), 24-26.

9. Laham, S. M., Koval, P., \& Alter, A. L. (2012). The name-pronunciation effect: Why people like Mr. Smith more than Mr. Colquhoun. Journal of Experimental Social Psychology, 48(3), 752-756.

10. Coffey, B., \& McLaughlin, P. A. (2009). Do masculine names help female lawyers become judges? Evidence from South Carolina. American Law and Economics Review, 11(1), 112-133.

11. Urbatsch, R. (2018). Feminine-sounding names and electoral performance. Electoral Studies, 55, 54-61.

12. Weber, M. (2018). The effects of listing authors in alphabetical order: A review of the empirical evidence. Research Evaluation, 27(3), 238-245.

13. Silberzahn, R., \& Uhlmann, E. L. (2013). It pays to be Herr Kaiser: Germans with noble-sounding surnames more often work as managers than as employees. Psychological Science, 24(12), 2437-2444.

14. Simonsohn, U. (2011a). Spurious? Name similarity effects (implicit egotism) in marriage, job, and moving decisions. Journal of Personality and Social Psychology, 101(1), 1-24.

15. Simonsohn, U. (2011b). Spurious also? Name-similarity effects (implicit egotism) in employment decisions. Psychological Science, 22(8), 1087-1089.

16. Sleigh, J. (2016). What's in a name? Nominative determinism in the UK dental workforce. British Dental Journal, 221(12), 773-776.

17. Abramo, G., \& D'Angelo, C. A. (2017). Does your surname affect the citability of your publications? Journal of Informetrics, 11(1), 121-127.

18. Yuret, T. (2019). A longitudinal analysis of the effect of alphabetization on academic careers. Data and Information Management, 3(2), 72-83.

19. Silberzahn, R., Simonsohn, U., \& Uhlmann, E. L. (2014). Matched-names analysis reveals no evidence of name-meaning effects: A collaborative commentary on Silberzahn and Uhlmann (2013). Psychological Science, 25(7), 1504-1505.

20. Gallucci, M. (2003). I sell seashells by the seashore and my name is Jack: Comment on Pelham, Mirenberg, and Jones (2002). Journal of Personality and Social Psychology, 85(5), 789-799. 
21. Lynn, M., \& Snyder, C. R. (2002). Uniqueness seeking. In C. R. Snyder \& S. J. Lopez (Eds.), Handbook of positive psychology (pp. 395-410). New York, NY: Oxford University Press.

22. Snyder, C. R., \& Fromkin, H. L. (1980). Uniqueness: The human pursuit of difference. New York, NY: Plenum.

23. Vignoles, V. L. (2011). Identity motives. In S. J. Schwartz, K. Luyckx, \& V. L. Vignoles (Eds.), Handbook of identity theory and research (pp. 403-432). New York, NY: Springer.

24. Becker, M., Vignoles, V. L., Owe, E., Brown, R., Smith, P. B., Easterbrook, M., .. Yamakoğlu, N. (2012). Culture and the distinctiveness motive: Constructing identity in individualistic and collectivistic contexts. Journal of Personality and Social Psychology, 102(4), 833-855.

25. Twenge, J. M., Abebe, E. M., \& Campbell, W. K. (2010). Fitting in or standing out: Trends in American parents' choices for children's names, 1880-2007. Social Psychological and Personality Science, 1(1), 19 25.

26. Ogihara, Y., Fujita, H., Tominaga, H., Ishigaki, S., Kashimoto, T., Takahashi, A., ...Uchida, Y. (2015). Are common names becoming less common? The rise in uniqueness and individualism in Japan. Frontiers in Psychology, 6, 1490.

27. Cai, H., Zou, X., Feng, Y., Liu, Y., \& Jing, Y. (2018). Increasing need for uniqueness in contemporary China: Empirical evidence. Frontiers in Psychology, 9, 554.

28. Cai, H., Huang, Z., \& Jing, Y. (2019). Living in a changing world: The change of culture and psychology. In D. Matsumoto \& H. C. Hwang (Eds.), The handbook of culture and psychology (2nd ed., pp. 786-817). New York, NY: Oxford University Press.

29. Jackson, J. C., Gelfand, M., De, S., \& Fox, A. (2019). The loosening of American culture over 200 years is associated with a creativity-order trade-off. Nature Human Behaviour, 3, 244-250.

30. Cotton, J., O'Neill, B. S., \& Griffin, A. (2008). The "name game": Affective and hiring reactions to first names. Journal of Managerial Psychology, 23(1), 18-39.

31. Pascual, A., Guéguen, N., Vallée, B., Lourel, M., \& Cosnefroy, O. (2015). First name popularity as predictor of employability. Names, 63(1), 30-36.

32. Zweigenhaft, R. L. (1977). The other side of unusual first names. Journal of Social Psychology, 103(2), 291-302.

33. Sadowski, C. J., Wheeler, K. J., \& Cash, M. (1983). Unusual first names and achievement among male psychologists. Journal of Social Psychology, 119(2), 181-185.

34. Holland, J. L. (1997). Making vocational choices: A theory of vocational personalities and work environments (3rd ed.). Odessa, FL: Psychological Assessment Resources.

35. Armstrong, P. I., Day, S. X., McVay, J. P., \& Rounds, J. (2008). Holland's RIASEC model as an integrative framework for individual differences. Journal of Counseling Psychology, 55(1), 1-18.

36. Su, R., Murdock, C., \& Rounds, J. (2015). Person-environment fit. In P. J. Hartung, M. L. Savickas, \& W. B. Walsh (Eds.), APA handbook of career intervention, Volume 1: Foundations (pp. 81-98). Washington, DC: American Psychological Association.

37. Cunningham, S. (2011). Developments in measuring the "creative" workforce. Cultural Trends, 20(1), 2540.

38. Gabe, T. M. (2006). Growth of creative occupations in U.S. metropolitan areas: A shift-share analysis. Growth and Change, 37(3), 396-415.

39. Roulin, N., Bangerter, A., \& Yerly, E. (2011). The uniqueness effect in selection interviews. Journal of 
Personnel Psychology, 10(1), 43-47.

40. Edwards, J. R., Caplan, R. D., \& Harrison, R. V. (1998). Person-environment fit theory: Conceptual foundations, empirical evidence, and directions for future research. In C. L. Cooper (Ed.), Theories of organizational stress (pp. 28-67). Oxford: Oxford University Press.

41. Kristof, A. L. (1996). Person-organization fit: An integrative review of its conceptualizations, measurement, and implications. Personnel Psychology, 49(1), 1-49.

42. Greguras, G. J., \& Diefendorff, J. M. (2009). Different fits satisfy different needs: Linking personenvironment fit to employee commitment and performance using self-determination theory. Journal of Applied Psychology, 94(2), 465-477.

43. Zweigenhaft, R. L. (1981). Unusual names and uniqueness. Journal of Social Psychology, 114(2), 297298.

44. Lynn, M., \& Harris, J. (1997). Individual differences in the pursuit of self-uniqueness through consumption. Journal of Applied Social Psychology, 27(21), 1861-1883.

45. Sen, S., Acar, S., \& Cetinkaya, C. (2014). Development of the Person-Environment Fit Scale (PEFSC): A new measure of creativity. Psychology of Aesthetics, Creativity, and the Arts, 8(4), 433-445.

46. Duan, W., Li, Y., Tang, X., Zhang, H., \& Cheng, X. (2019). Determination of the classification role of Person-Environment Fit Scale for Creativity in workplace context. Creativity Research Journal, 31(2), 198-206.

47. Kristof-Brown, A. L., Zimmerman, R. D., \& Johnson, E. C. (2005). Consequences of individuals' fit at work: A meta-analysis of person-job, person-organization, person-group, and person-supervisor fit. Personnel Psychology, 58, 281-342.

48. Oliver, J. E., Wood, T., \& Bass, A. (2016). Liberellas versus Konservatives: Social status, ideology, and birth names in the United States. Political Behavior, 38(1), 55-81.

49. Strobl, C., Malley, J., \& Tutz, G. (2009). An introduction to recursive partitioning: Rationale, application, and characteristics of classification and regression trees, bagging, and random forests. Psychological Methods, 14(4), 323-348.

50. Zhu, B., \& Millward, C. (1987). Personal names in Chinese. Names, 35(1), 8-21.

51. Atir, S., \& Ferguson, M. J. (2018). How gender determines the way we speak about professionals. Proceedings of the National Academy of Sciences, 115(28), 7278-7283.

52. Hirsch, J. E. (2007). Does the $h$ index have predictive power? Proceedings of the National Academy of Sciences, 104(49), 19193-19198.

53. Zwebner, Y., Sellier, A.-L., Rosenfeld, N., Goldenberg, J., \& Mayo, R. (2017). We look like our names: The manifestation of name stereotypes in facial appearance. Journal of Personality and Social Psychology, $112(4), 527-554$.

54. Şimşek, Ö. F., \& Yalınçetin, B. (2010). I feel unique, therefore I am: The development and preliminary validation of the personal sense of uniqueness (PSU) scale. Personality and Individual Differences, 49(6), 576-581.

55. Hox, J. J., Moerbeek, M., \& van de Schoot, R. (2018). Multilevel analysis: Techniques and applications (3rd ed.). New York, NY: Routledge. 


\section{Tables}

Table 1. Overview of the present studies.

\begin{tabular}{|c|c|c|c|c|c|}
\hline Study & Outcome variables & Method & Country & Sample size & Demographic control variables \\
\hline Study 1 & $\begin{array}{l}\text { Future job choice (i.e., } \\
\text { career planning) }\end{array}$ & Survey & China & 672 & $\begin{array}{l}\text { Gender, age, expected highest } \\
\text { education, family income, } \\
\text { parents' education, parents' } \\
\text { occupations }\end{array}$ \\
\hline Study 2 & Hypothetical job choice & Experiment & China & 1,048 & $\begin{array}{l}\text { Gender, age, education, } \\
\text { parents' education }\end{array}$ \\
\hline Study 3 & Real job choice & $\begin{array}{l}\text { Big data } \\
\text { (resume) }\end{array}$ & China & $2,480,269$ & Gender, age, education \\
\hline Study 4 & Real job choice & $\begin{array}{l}\text { Big data } \\
\text { (resume) }\end{array}$ & U.S. \& U.K. & $1,030,504$ & Gender \\
\hline Study 5 & $\begin{array}{l}\text { Job-hunting intention } \\
\text { Potential achievement }\end{array}$ & $\begin{array}{l}\text { Big data } \\
\text { (resume) }\end{array}$ & China & $3,134,813$ & $\begin{array}{l}\text { Gender, age, education, } \\
\text { working experience, city }\end{array}$ \\
\hline Study 6 & $\begin{array}{l}\text { Real job choice } \\
\text { Real achievement }\end{array}$ & $\begin{array}{l}\text { Big data (web } \\
\text { archive) }\end{array}$ & China & 673,387 & $\begin{array}{l}\text { Most with gender and/or age } \\
\text { (depending on data sources) }\end{array}$ \\
\hline Study 7 & Real achievement & $\begin{array}{l}\text { Big data (web } \\
\text { archive) }\end{array}$ & U.S. \& U.K. & 57,791 & $\begin{array}{l}\text { Most with gender and/or age } \\
\text { (depending on data sources) }\end{array}$ \\
\hline
\end{tabular}

Note. Studies 1-6 examined the name-job uniqueness fit (H1). Studies 1-2 also examined its possible mechanisms (H2). Studies 5-7 further examined its potential benefit to career achievement (H3). As shown in this table, we addressed alternative explanations such as family SES and birth cohort in most studies. In Supplementary Studies S1 and S2, we ruled out another alternative explanation — parents' need for uniqueness - underlying the name-job uniqueness fit. 
Table 2. Name uniqueness predicts the uniqueness of future jobs (Study 1, OLS regression).

\begin{tabular}{|c|c|c|c|c|}
\hline \multirow[b]{2}{*}{ Predictor } & \multicolumn{2}{|c|}{ Job uniqueness (obj.) } & \multicolumn{2}{|c|}{ Job uniqueness (subj.) } \\
\hline & $\beta$ & $95 \% \mathrm{CI}$ & $\beta$ & $95 \% \mathrm{CI}$ \\
\hline \multicolumn{5}{|l|}{ Model 1.1/1.2 } \\
\hline Given-name uniqueness (obj.) & $0.143 * * *$ & {$[0.068,0.218]$} & - & - \\
\hline Given-name uniqueness (subj.) & - & - & $0.178 * * *$ & {$[0.103,0.253]$} \\
\hline$R^{2}$ & & $2.0 \%$ & & $3.2 \%$ \\
\hline \multicolumn{5}{|l|}{ Model 2.1/2.2 } \\
\hline Given-name uniqueness (obj.) & $0.139 * * *$ & {$[0.064,0.213]$} & - & - \\
\hline Given-name uniqueness (subj.) & - & - & $0.116 * *$ & {$[0.044,0.187]$} \\
\hline Need for uniqueness (NfU) & $0.135 * * *$ & {$[0.061,0.210]$} & $0.328 * * *$ & {$[0.256,0.400]$} \\
\hline$R^{2}$ & & $3.9 \%$ & & $13.5 \%$ \\
\hline \multicolumn{5}{|l|}{ Model 3.1/3.2 } \\
\hline Given-name uniqueness (obj.) & $0.158 * *$ & {$[0.052,0.263]$} & - & - \\
\hline Given-name uniqueness (subj.) & - & - & $0.117 * *$ & {$[0.042,0.192]$} \\
\hline Character uniqueness in corpus & -0.016 & {$[-0.103,0.070]$} & -0.040 & {$[-0.113,0.034]$} \\
\hline Given-name valence & $0.093^{\dagger}$ & {$[-0.003,0.190]$} & $0.083 *$ & {$[0.011,0.155]$} \\
\hline Given-name gender & -0.060 & {$[-0.157,0.037]$} & $-0.079^{\dagger}$ & {$[-0.171,0.014]$} \\
\hline Need for uniqueness (NfU) & $0.115 * *$ & {$[0.036,0.194]$} & $0.285 * * *$ & {$[0.207,0.362]$} \\
\hline Sense of uniqueness (SoU) & 0.026 & {$[-0.052,0.105]$} & $0.093 *$ & {$[0.015,0.171]$} \\
\hline $\begin{array}{c}\text { Data source }(0=\text { WJX.cn, } \\
1=\text { university sample })\end{array}$ & $0.140 * *$ & {$[0.052,0.229]$} & $0.093 *$ & {$[0.006,0.179]$} \\
\hline Gender $(0=$ female, $1=$ male $)$ & 0.067 & {$[-0.030,0.164]$} & $0.117^{*}$ & {$[0.021,0.213]$} \\
\hline Age & $-0.082^{\dagger}$ & {$[-0.165,0.001]$} & 0.062 & {$[-0.020,0.143]$} \\
\hline Expected highest education & $0.204 * * *$ & {$[0.131,0.277]$} & -0.004 & {$[-0.076,0.067]$} \\
\hline Family income & -0.027 & {$[-0.112,0.058]$} & 0.064 & {$[-0.020,0.147]$} \\
\hline Parents' education & -0.004 & {$[-0.093,0.085]$} & -0.020 & {$[-0.108,0.067]$} \\
\hline Parents' job uniqueness (obj.) & $0.082^{\dagger}$ & {$[-0.005,0.169]$} & -0.061 & {$[-0.147,0.024]$} \\
\hline$R^{2}$ & & $13.7 \%$ & & $17.0 \%$ \\
\hline
\end{tabular}

Note. $N=672$. Standardized regression coefficients $(\beta)$ and $95 \%$ CIs are presented. Obj. $=$ objective index. Subj. = subjective rating. Surnames were not available in Study 1. For details about the computation of name variables, see Supplementary Method 2.

${ }^{\dagger} P<0.10, * P<0.05, * * P<0.01, * * * P<0.001$. 
Table 3. Experiencing holding unique given names increases need for uniqueness and the possibility to choose unique jobs (Study 2, OLS and logistic regression).

\begin{tabular}{|c|c|c|c|c|}
\hline \multirow[b]{2}{*}{ Predictor } & \multicolumn{2}{|c|}{ Need for uniqueness } & \multicolumn{2}{|c|}{ Unique (vs. common) job } \\
\hline & $B_{\mathrm{OLS}}$ & $95 \% \mathrm{CI}$ & $B_{\text {logistic }}$ & $95 \% \mathrm{CI}$ \\
\hline \multicolumn{5}{|l|}{ Study 2a: Model 1.1/1.2 } \\
\hline Unique (vs. common) name & $0.135^{*}$ & {$[0.005,0.264]$} & $0.490 * *$ & {$[0.138,0.843]$} \\
\hline Gender & 0.036 & {$[-0.093,0.165]$} & $0.417^{*}$ & {$[0.065,0.770]$} \\
\hline Name $\times$ Gender & 0.052 & {$[-0.207,0.310]$} & 0.094 & {$[-0.611,0.799]$} \\
\hline$R^{2}$ & & $0.8 \%$ & & $3.1 \%$ \\
\hline \multicolumn{5}{|l|}{ Study 2b: Model 2.1/2.2 } \\
\hline Unique (vs. common) name & $0.321 * * *$ & {$[0.180,0.462]$} & $0.597 * *$ & {$[0.231,0.964]$} \\
\hline Gender & 0.085 & {$[-0.056,0.226]$} & 0.199 & {$[-0.167,0.565]$} \\
\hline Name $\times$ Gender & -0.112 & {$[-0.394,0.170]$} & -0.064 & {$[-0.797,0.669]$} \\
\hline$R^{2}$ & & $4.5 \%$ & & $3.2 \%$ \\
\hline \multicolumn{5}{|l|}{ Combined: Model 3.1/3.2 } \\
\hline Unique (vs. common) name & $0.219 * * *$ & {$[0.123,0.314]$} & $0.522 * * *$ & {$[0.271,0.772]$} \\
\hline Gender & 0.060 & {$[-0.035,0.155]$} & $0.308^{*}$ & {$[0.057,0.558]$} \\
\hline Name $\times$ Gender & -0.023 & {$[-0.213,0.168]$} & 0.046 & {$[-0.455,0.547]$} \\
\hline$R^{2}$ & & $2.1 \%$ & & $2.9 \%$ \\
\hline \multicolumn{5}{|l|}{ Combined: Model 4.1/4.2 } \\
\hline Unique (vs. common) name & $0.230 * * *$ & {$[0.134,0.326]$} & $0.429 * *$ & {$[0.166,0.693]$} \\
\hline Gender & 0.061 & {$[-0.037,0.159]$} & $0.238^{\dagger}$ & {$[-0.031,0.508]$} \\
\hline Study $(2 a / 2 b)$ & -0.017 & {$[-0.113,0.079]$} & $0.688 * * *$ & {$[0.423,0.953]$} \\
\hline Name $\times$ Gender & -0.028 & {$[-0.219,0.163]$} & 0.033 & {$[-0.493,0.558]$} \\
\hline Name $\times$ Study & $0.187^{\dagger}$ & {$[-0.004,0.378]$} & -0.012 & {$[-0.539,0.515]$} \\
\hline Gender $\times$ Study & 0.038 & {$[-0.154,0.231]$} & -0.289 & {$[-0.818,0.240]$} \\
\hline Name $\times$ Gender $\times$ Study & -0.176 & {$[-0.560,0.208]$} & -0.029 & {$[-1.084,1.027]$} \\
\hline Need for uniqueness (NfU) & - & - & $0.665 * * *$ & {$[0.441,0.888]$} \\
\hline Sense of uniqueness (SoU) & - & - & 0.060 & {$[-0.190,0.309]$} \\
\hline Surname uniqueness & 0.035 & {$[-0.027,0.098]$} & 0.007 & {$[-0.164,0.177]$} \\
\hline Age & 0.021 & {$[-0.010,0.053]$} & 0.049 & {$[-0.038,0.137]$} \\
\hline Education & $-0.119^{*}$ & {$[-0.229,-0.010]$} & 0.029 & {$[-0.272,0.330]$} \\
\hline Parents' education & 0.036 & {$[-0.026,0.099]$} & 0.059 & {$[-0.111,0.229]$} \\
\hline$R^{2}$ & & $3.1 \%$ & & $14.0 \%$ \\
\hline
\end{tabular}

Note. Experimental conditions were effect-coded: (i) name manipulation $(-0.5=$ common, $0.5=$ unique $)$, (ii) participant gender $(-0.5=$ female, $0.5=$ male; same as the new identity's gender), and (iii) study $(-0.5$ $=2 \mathrm{a}, 0.5=2 \mathrm{~b})$. Study $2 \mathrm{a}(N=578)$ specified the major of the new identity as 'economy' (a popular, common major); Study $2 \mathrm{~b}(N=470)$ did not specify the major of the new identity. Unstandardized regression coefficients $(B)$ and $95 \%$ CIs are reported for meaningful interpretation of the effect-coded variables. For logistic regression, we report Nagelkerke's $R^{2}$.

$\dagger P<0.10, * P<0.05, * * P<0.01, * * * P<0.001$. 


\section{Figures}
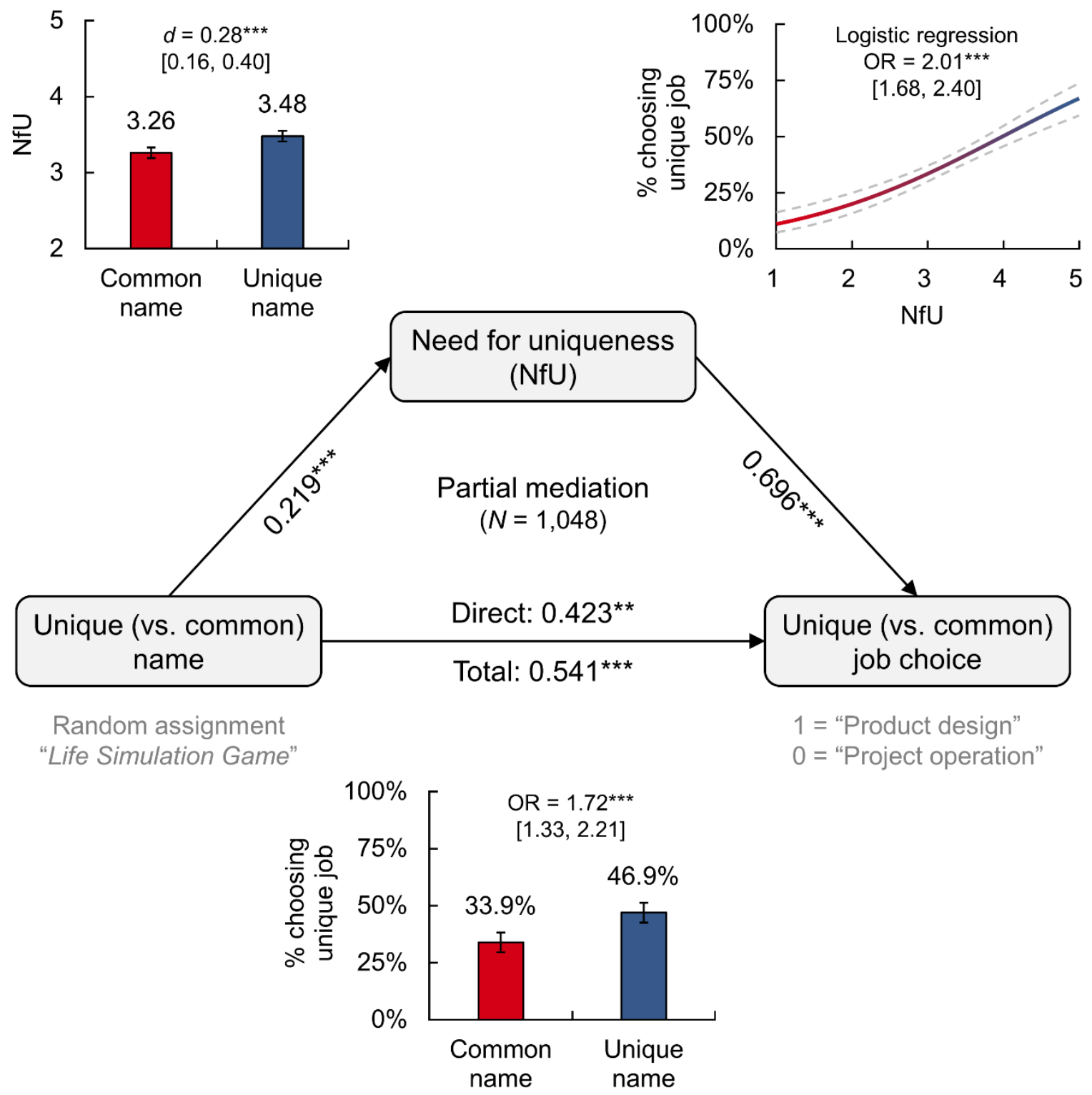

Fig. 1. Experiencing holding unique given names increases the possibility to choose unique jobs through increasing need for uniqueness (Study 2).

Unstandardized path coefficients are displayed. The main effects of gender and study version $(2 \mathrm{a} / 2 \mathrm{~b})$ were controlled in all paths. Error bars/bands represent $95 \%$ confidence interval.

$* P<0.05,{ }^{*} P<0.01, * * * P<0.001$. 

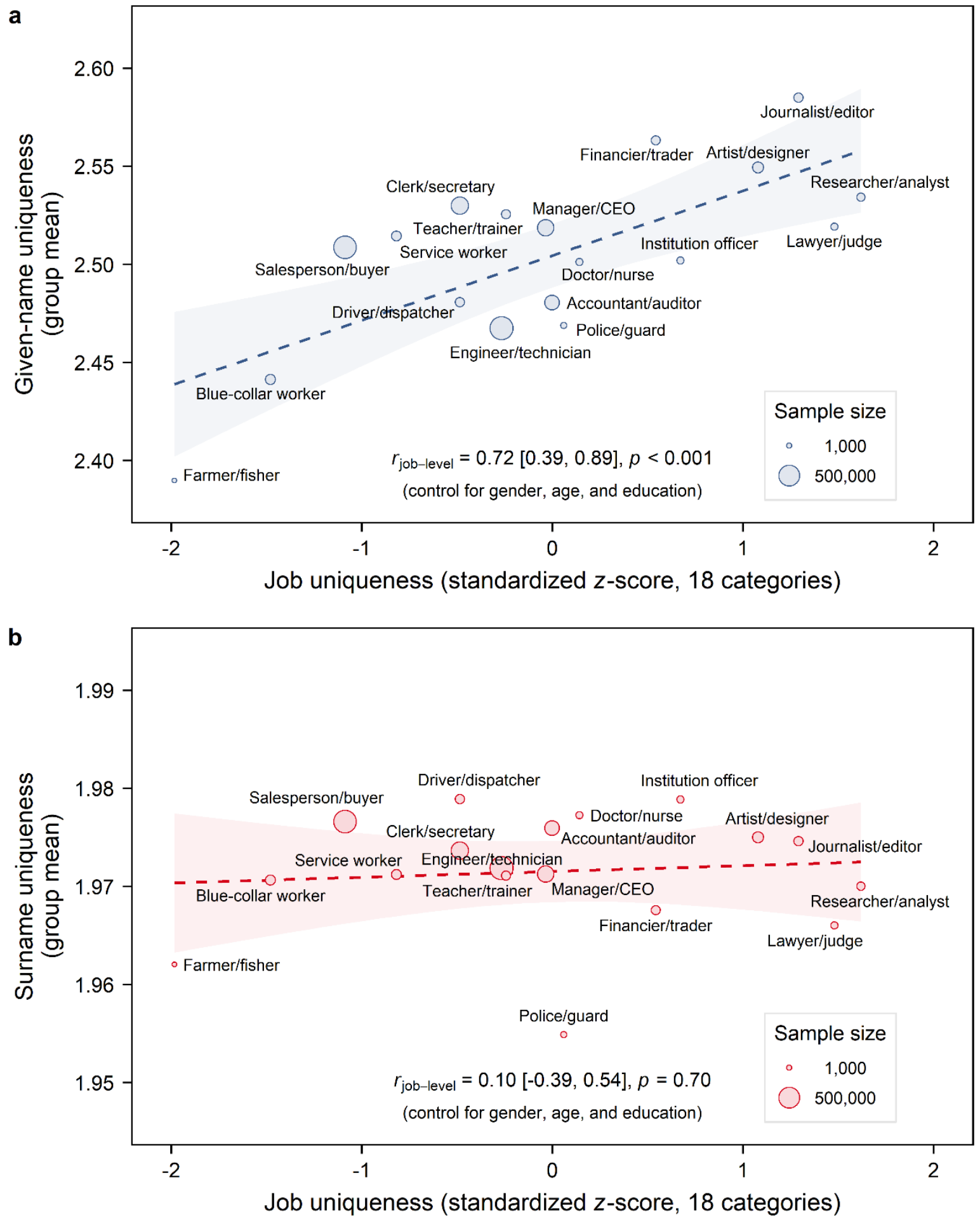

Fig. 2. Chinese workers in more unique jobs hold more unique given names but not more unique surnames (Study 3).

Job-level partial correlations and regression lines are displayed. Marginal group means were adjusted for all available demographics. Error bands represent 95\% confidence interval. 


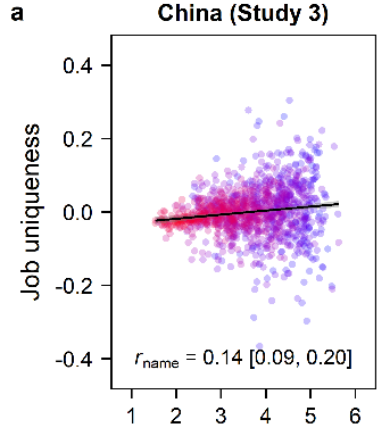

Given-name uniqueness

$(1,185$ single characters)

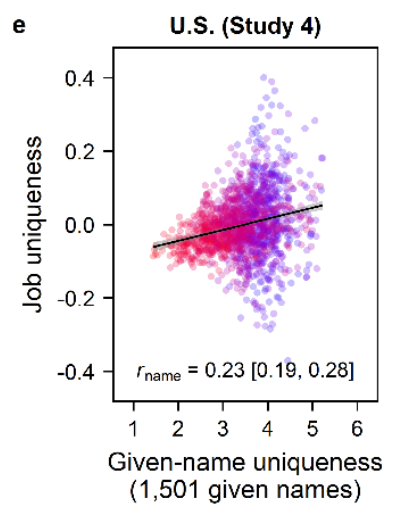

i

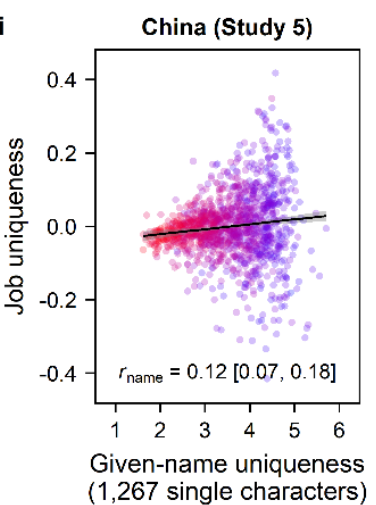

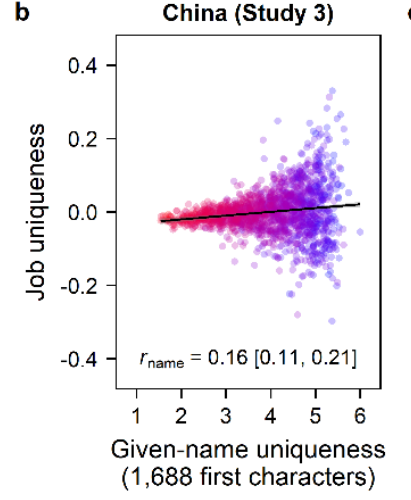
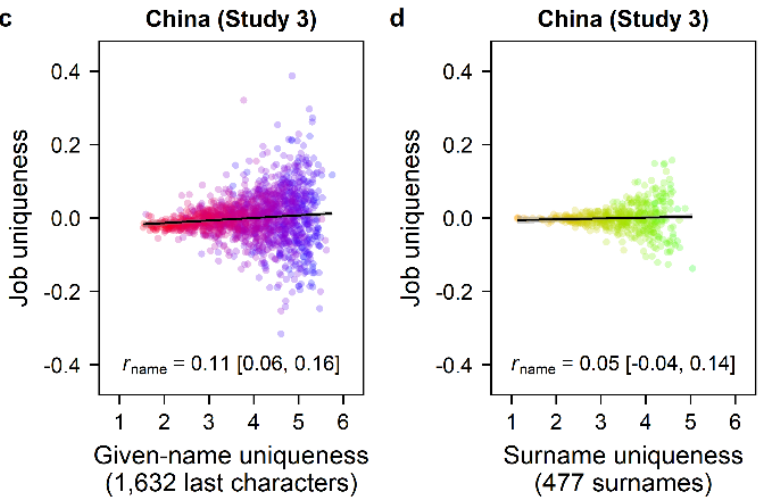

f U.K. (Study 4) g
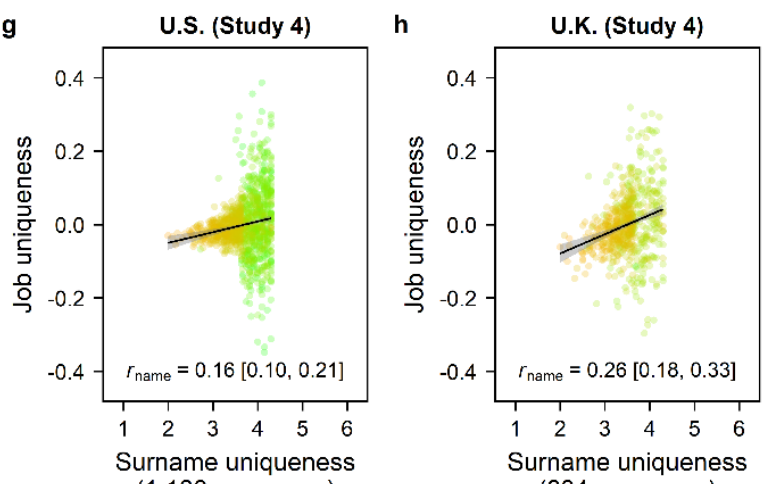

$(1,130$ surnames $)$

k

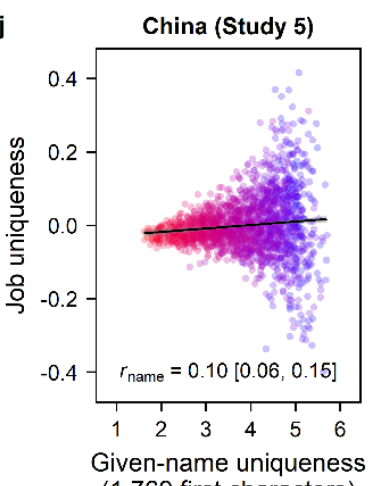

$(1,769$ first characters $)$
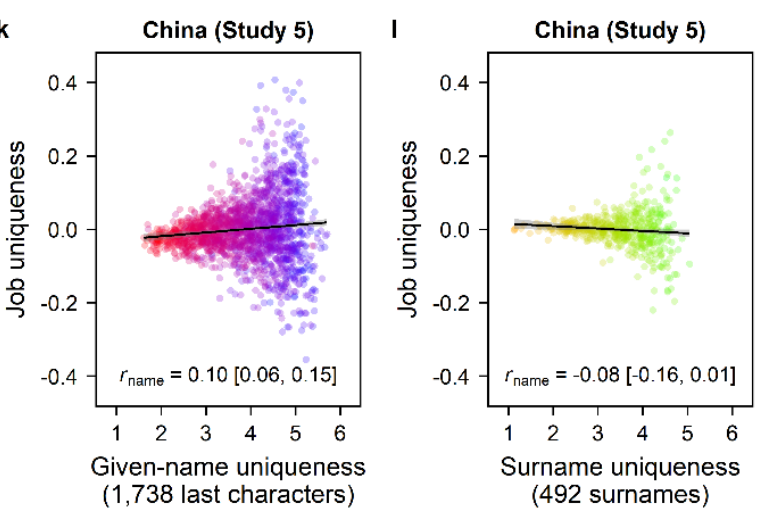

Fig. 3. Name uniqueness predicts job uniqueness among employees (Studies 3-4) and job hunters (Study 5) in real life.

Name-level partial correlations and regression lines are displayed. Marginal group means were adjusted for all available demographics. Each red/blue dot represents a given name or given-name character; given names of larger (smaller) sample sizes are denoted in red (blue). Each orange/green dot represents a surname; surnames of larger (smaller) sample sizes are denoted in orange (green). For comparison, job uniqueness in this figure was centered at the name level $(0=$ averaged job uniqueness across names). Error bands represent $95 \%$ confidence interval. 

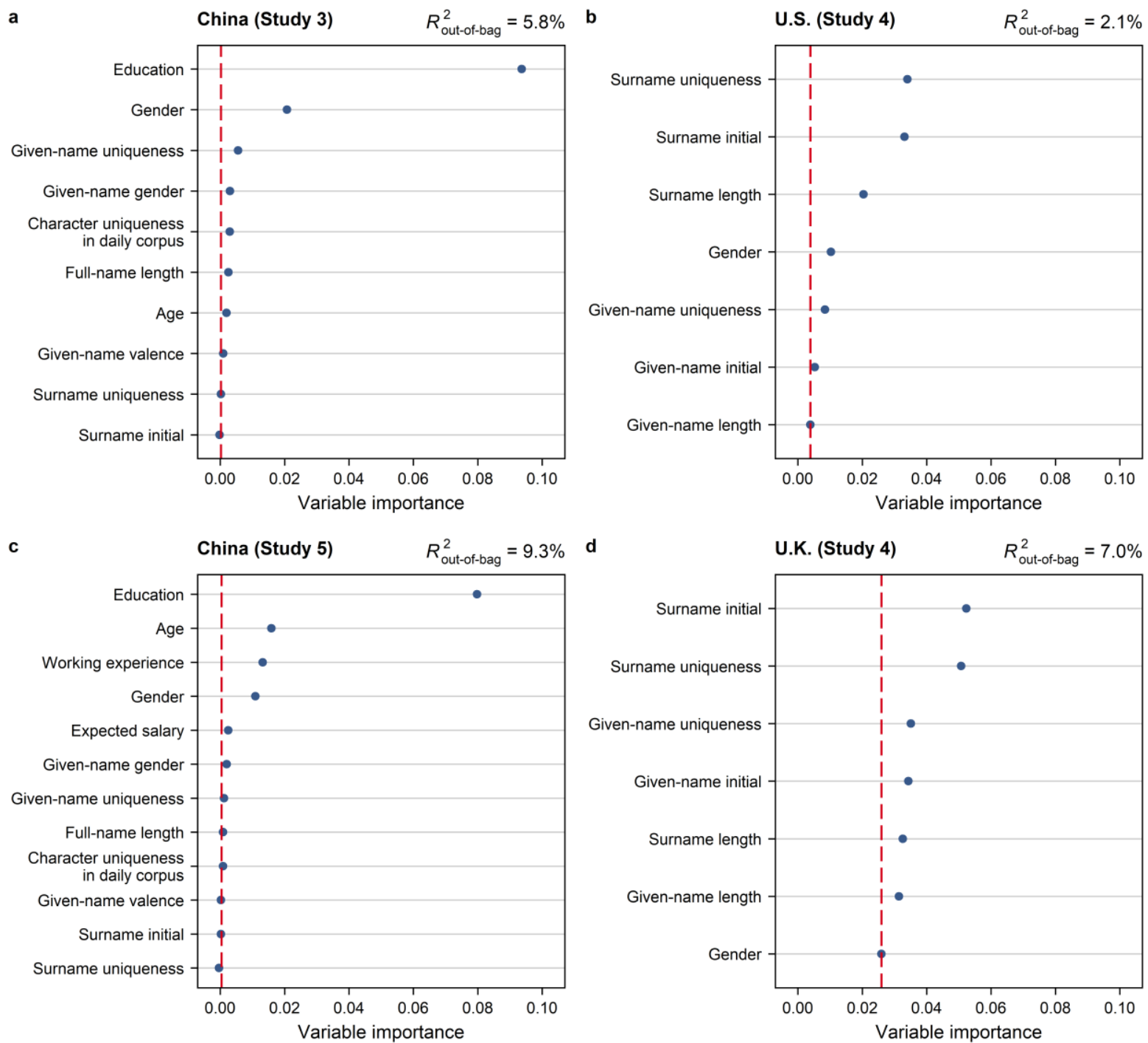

Fig. 4. Variable importance in machine learning for predicting job uniqueness (Studies 3-5).

Variables exceeding the red dashed line (threshold of random noise) are relatively important predictors. Each plot presents the average scores obtained from 10 randomly selected subsamples (each $n=10,000$ ) with balanced group sizes across job categories. $R^{2}$ out-of-bag $=$ prediction accuracy on the testing dataset by the Conditional Random Forest algorithm. For details, see Supplementary Method 3. 


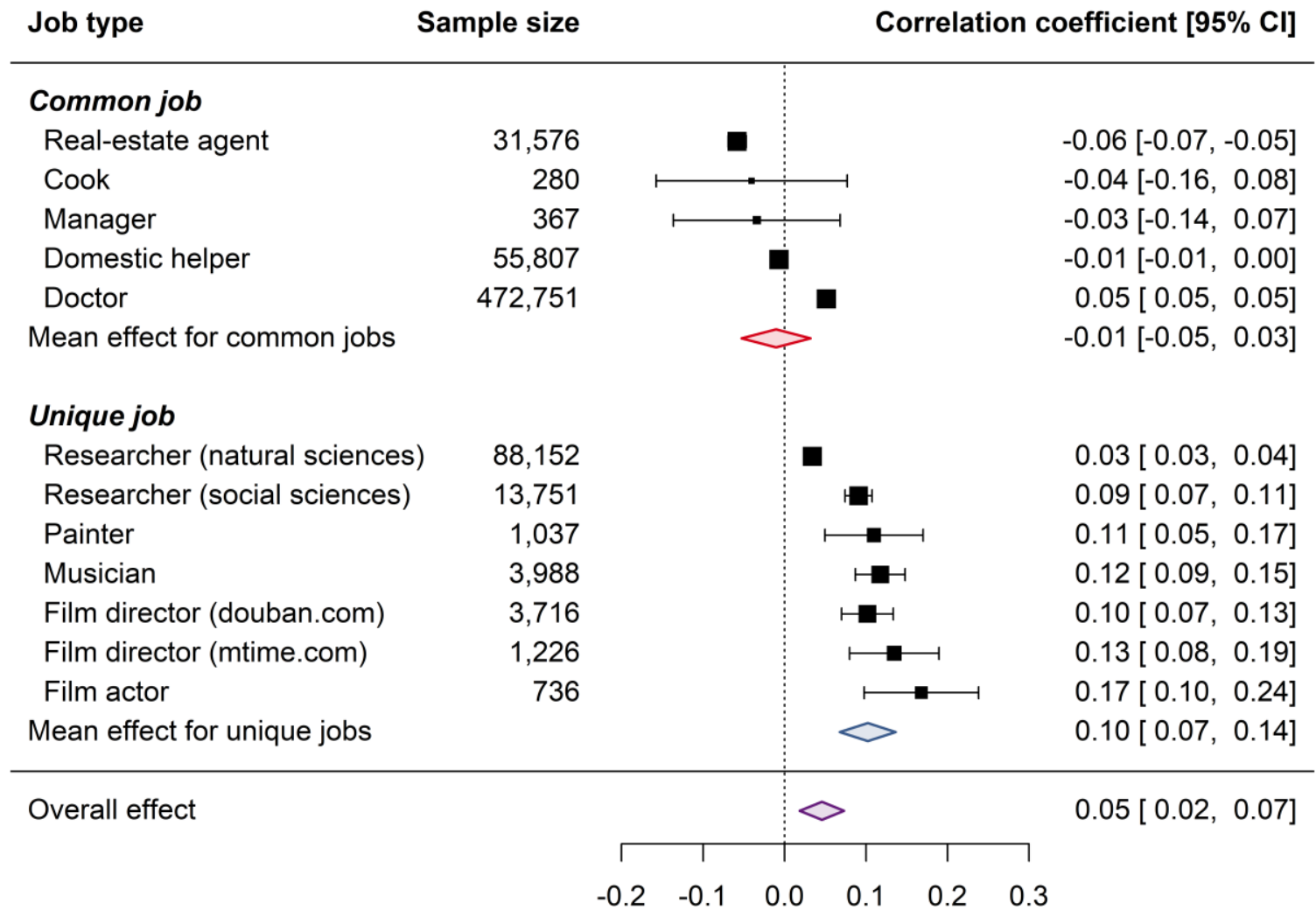

Correlation $(r)$ between given-name uniqueness and career achievement

Fig. 5. Given-name uniqueness is positively correlated with career achievement in unique jobs but not with achievement in common jobs (Study 6).

The size of black squares relates to sample size. Red, blue, and purple rhombuses display mean estimate and 95\% confidence interval of Pearson's $r$ for common jobs, unique jobs, and all jobs, respectively. For details about data sources and career-achievement indices, see Supplementary Table 10. 